\title{
Kadec-Klee properties of some quasi-Banach function spaces
}

\author{
Pawel Kolwicz ${ }^{1}$
}

Received: 20 October 2017 / Accepted: 10 January 2018 / Published online: 22 January 2018

(C) The Author(s) 2018. This article is an open access publication

\begin{abstract}
We study Kadec-Klee properties with respect to global (local) convergence in measure in quasi-Banach function spaces. First, we prove some general results which can be of independent interest. Next, we investigate these properties in symmetrizations $E^{(*)}$. Finally, we apply general results to study these properties in Marcinkiewicz and Lorentz spaces.
\end{abstract}

Keywords Symmetric spaces - Symmetrization of the quasi-Banach function space Lorentz space $\cdot$ Marcinkiewicz space $\cdot$ Kadec-Klee properties $\cdot$ Local structure of a separated point

Mathematics Subject Classification 46E30 - 46B20 • 46B42

\section{Introduction}

Geometry of Banach spaces has found a lot of applications and has been intensively developed during the last decades. However, the studies of global properties are not always sufficient. The geometric structure of a separated point of a Banach space (Banach lattice) has been intensively investigated recently (see [7, 8, 14, 15, 19, 24, 25, $27,28])$. On the other hand, a symmetrization $E^{(*)}$ of a quasi-Banach function space $E$ is an important construction covering several classical classes of Banach function spaces (see [12,13,21,22,24-26]).

Paweł Kolwicz

pawel.kolwicz@put.poznan.pl

1 Institute of Mathematics, Faculty of Electrical Engineering, Poznań University of Technology, Piotrowo 3A, 60-965 Poznan, Poland 
We study Kadec-Klee properties with respect to global (local) convergence in measure. We concentrate on the structure of the separated point. In Sect. 2 we recall the necessary terminology. In Sect. 3 we prove some general results concerning quasiBanach function spaces which can be of independent interest (the most interesting and useful in this section are Lemma 3.2, Theorems 3.9, 3.15 and 3.16). Obviously, studying the structure of quasi-normed function spaces, instead of normed function spaces, often forces the need to use new methods. Next, in Sect. 4, we investigate Kadec-Klee properties in symmetrizations $E^{(*)}$. The natural way is to express properties of $E^{(*)}$ by the respective properties of $E$ (see Theorem 4.9). We discuss also whether some assumptions of Theorem 4.9 are essential and we show that assumptions of Theorem 4.9(i) are independent. It is also worth to mention Proposition 4.14 which gives a new range of application for some useful characterization of convergence $x_{n} \rightarrow x$ in norm. Finally, in Sects. 5 and 6, we apply our general results to characterize the local structure in Marcinkiewicz and Lorentz spaces. Recall also that the structure of a separated point has applications in the local best dominated approximation problems in Banach lattices (see $[7,8])$.

\section{Preliminaries}

Given a real vector space $X$ the functional $x \mapsto\|x\|$ is called a quasi-norm if the following three conditions are satisfied:

(i) $\|x\|=0$ if and only if $x=0$;

(ii) $\|a x\|=|a|\|x\|, x \in X, a \in R$;

(iii) there exists $C=C_{X} \geq 1$ such that $\|x+y\| \leq C(\|x\|+\|y\|)$ for all $x, y \in X$.

We call $\|\cdot\|$ a $p$-norm where $0<p \leq 1$ if, in addition, it is $p$-subadditive, that is, $\|x+y\|^{p} \leq\|x\|^{p}+\|y\|^{p}$ for all $x, y \in X$.

By the Aoki-Rolewicz theorem (cf. [17, Theorem 1.3 on p. 7] , [32, p. 86]), if $0<p \leq 1$ is such that $C=2^{1 / p-1}$, then there exists a $p$-norm $\|\cdot\|_{1}$ which is equivalent to $\|\cdot\|$ so that

$$
\|x+y\|_{1}^{p} \leq\|x\|_{1}^{p}+\|y\|_{1}^{p} \text { and }\|x\|_{1} \leq\|x\| \leq 2 C\|x\|_{1}
$$

for all $x, y \in X$. The quasi-norm $\|\cdot\|$ induces a metric topology on $X$ : in fact, a metric can be defined by $d(x, y)=\|x-y\|_{1}^{p}$, when the quasi-norm $\|\cdot\|_{1}$ is $p$-subadditive. We say that $X=(X,\|\cdot\|)$ is a quasi-Banach space if it is complete for this metric.

As usual $S(X)$ (resp. $B(X)$ ) stands for the unit sphere (resp. the closed unit ball) of a real quasi-Banach space $\left(X,\|\cdot\|_{X}\right)$.

We denote by $L^{0}$ the set of all (equivalence classes of) extended real valued Lebesgue measurable functions on $I=(0, \alpha)$, where $\alpha=1$ or $\alpha=\infty$. Let $m$ be the Lebesgue measure on $(0, \alpha)$.

A quasi-Banach lattice $\left(E,\|\cdot\|_{E}\right)$ is called a quasi-Banach function space (or a quasi-Köthe space) if it is a linear subspace of $L^{0}$ satisfying the following conditions:

(1) If $x \in L^{0}, y \in E$ and $|x| \leq|y| m$-a.e., then $x \in E$ and $\|x\|_{E} \leq\|y\|_{E}$.

(2) There exists a strictly positive $x \in E$. 
By $E_{+}$we denote the positive cone of $E$, that is, $E_{+}=\{x \in E: x \geq 0\}$. For $x \in L^{0}$ set

$$
\operatorname{supp} x=\{t \in I: x(t) \neq 0\} .
$$

Moreover, let $E(w)$ be the weighted quasi-Banach function space, that is

$$
E(w)=\left\{x \in L^{0}: x w \in E\right\} \text { with the norm }\|x\|_{E(w)}=\|x w\|_{E}
$$

where $w: I \rightarrow[0, \infty)$ is a measurable weight function.

Moreover, the $p$-convexification $E^{(p)}$ of $E$, for $1<p<\infty$, is defined by

$$
E^{(p)}=\left\{x \in L^{0}:|x|^{p} \in E\right\} \text { and }\|x\|_{E^{(p)}}=\left\||x|^{p}\right\|_{E}^{1 / p} .
$$

If $0<p<1$, we say about $p$-concavification $E^{(p)}$ of $E$.

For $x \in L^{0}$, its distribution function is defined by

$$
d_{x}(\lambda)=m\{s \in[0, \alpha):|x(s)|>\lambda\}, \quad \lambda \geq 0,
$$

and its decreasing rearrangement by

$$
x^{*}(t)=\inf \left\{\lambda>0: d_{x}(\lambda) \leq t\right\}, \quad t \geq 0
$$

Set $x^{*}(\infty)=\lim _{t \rightarrow \infty} x^{*}(t)$ if $I=(0, \infty)$ and $x^{*}(\infty)=0$ if $I=(0,1)$. For the properties of $d_{x}$ and $x^{*}$, the reader is referred to [3,29].

Two functions $x, y \in L^{0}$ are called equimeasurable ( $x \sim y$ for short) if $d_{x}=d_{y}$. We say that a quasi-normed function space $\left(E,\|\cdot\|_{E}\right)$ is rearrangement invariant (r.i. for short) or symmetric if whenever $x \in L^{0}$ and $y \in E$ with $x \sim y$, then $x \in E$ and $\|x\|_{E}=\|y\|_{E}$.

For any symmetric quasi-Banach function space $\left(E,\|\cdot\|_{E}\right)$ we have

$$
L^{p}(I) \cap L^{\infty}(I) \stackrel{C_{1}}{\hookrightarrow} E \stackrel{C_{2}}{\hookrightarrow} L^{p, \infty}(I)+L^{\infty}(I),
$$

where $C$ is from the $C$-triangle inequality for $\|\cdot\|_{E}$, the number $p$ satisfies the equality $C=2^{1 / p-1}, C_{1}=2^{1 / p}\left\|\chi_{(0,1)}\right\|_{E}, C_{2}=\frac{4^{1 / p}}{\left\|\chi_{(0,1)}\right\|_{E}}$ and $L^{p, \infty}=\left\{x \in L^{0}(I):\|x\|=\right.$ $\left.\sup _{t \in I} t^{1 / p} x^{*}(t)<\infty\right\}$ (see Theorem 1 and 3 from [2]). For more details about symmetric (quasi-)Banach function spaces see [3,20-22, 26, 29,32].

Recall that a quasi-Banach function space $E$ is called order continuous $(E \in(O C)$ ) if for each sequence $x_{n} \downarrow 0$, that is $x_{n} \geq x_{n+1}$ and $\inf _{n} x_{n}=0$, we have $\left\|x_{n}\right\|_{E} \rightarrow 0$ (see [18,31,35]). Moreover, $E \in(O C)$ if and only if for every element $x \in E$ and each sequence $\left(x_{n}\right)$ in $E$ satisfying conditions inf $\left\{x_{n}, x_{m}\right\}=0$ for $n \neq m$ and $0 \leq x_{n} \leq|x|$ we have $\left\|x_{n}\right\|_{E} \rightarrow 0$. The sufficiency follows from Proposition 2.2 in [30]. We prove the necessity. If the condition is not satisfied then we find an element $x \in E$ and a sequence $\left(x_{n}\right)$ in $E$ such that inf $\left\{x_{n}, x_{m}\right\}=0$ for $n \neq m, 0 \leq x_{n} \leq|x|$ and $\left\|x_{n}\right\|_{E} \nrightarrow 0$. Letting $y_{n}=\sum_{k=1}^{n} x_{k}$ and $y=\sum_{k=1}^{\infty} x_{k}$ we get $y-y_{n} \downarrow 0$ and $\left\|y-y_{n}\right\| \nrightarrow 0$, which means $E \notin(O C)$. 
The following equivalent conditions are well known for a Banach function space. We collect them (with a short explanation) in a case of quasi-Banach function space $E$ for further convenience.

Theorem 2.1 Let $E$ be a quasi-Banach function space and $x \in E$. The following statements are equivalent:

(i) For every sequence $\left(x_{n}\right)$ in E satisfying conditions inf $\left\{x_{n}, x_{m}\right\}=0$ for $n \neq m$ and $0 \leq x_{n} \leq|x|$ we have $\left\|x_{n}\right\|_{E} \rightarrow 0$.

(ii) For each sequence $\left(x_{n}\right)$ in $E$ such that $0 \leq x_{n} \leq|x|$ and $x_{n} \rightarrow 0 m$-a.e. we have $\left\|x_{n}\right\|_{E} \rightarrow 0$.

(iii) For any sequence $\left(x_{n}\right)$ in $E$ such that $0 \leq x_{n} \leq|x|$ and $x_{n} \rightarrow 0$ locally in measure we have $\left\|x_{n}\right\|_{E} \rightarrow 0$.

Proof The implication (ii) $\Rightarrow$ (i) is clear. The implication (i) $\Rightarrow$ (ii) can be proved as Lemma 5 in [15] (the proof works almost the same for a quasi-Banach function space). The implication (iii) $\Rightarrow$ (ii) is obvious. The implication (ii) $\Rightarrow$ (iii). Note that if $x_{n} \rightarrow 0$ locally in measure then $x_{n_{k}} \rightarrow 0$ a.e. for some subsequence $\left(x_{n_{k}}\right)$ of $\left(x_{n}\right)$, because the measure space is $\sigma$-finite. Applying the double extract subsequence principle we can finish the proof.

The author would like to thank Professor Witold Wnuk for the fruitful discussion concerning the above theorem, especially for pointing out that the equivalence (i) $\Leftrightarrow$ (iii) follows also from a general, deep result from the theory of locally solid Riesz spaces (see [1, Theorem 12.9]).

Theorem 2.1 gives a natural motivation for the following definition. A point $x \in E$ is said to have an order continuous norm ( $x$ is a point of order continuity, $x$ is an $O C$ point briefly) if for any sequence $\left(x_{n}\right)$ in $E$ such that $0 \leq x_{n} \leq|x|$ and $x_{n} \rightarrow 0$ $m$-a.e. we have $\left\|x_{n}\right\|_{E} \rightarrow 0$. Consequently, $E \in(O C)$ if and only if every element $x$ in $E$ has an order continuous norm. The symbol $E_{a}$ stands for the subspace of order continuous elements of $E$.

We assume in the paper (unless it is stated otherwise) that $E$ has the Fatou property, that is, if $0 \leq x_{n} \uparrow x \in L^{0}$ with $\left(x_{n}\right)_{n=1}^{\infty}$ in $E$ and $\sup _{n \in N}\left\|x_{n}\right\|_{E}<\infty$, then $x \in E$ and $\lim _{n}\left\|x_{n}\right\|_{E}=\|x\|_{E}$. Recall that in the definition of a semi-Fatou property we assume additionally that $x \in E$.

A point $x \in E$ is said to be an $H_{g}$-point (resp. $H_{l}$-point) in $E$ if for any $\left(x_{n}\right) \subset E$ such that $x_{n} \rightarrow x$ globally (resp. locally) in measure and $\left\|x_{n}\right\|_{E} \rightarrow\|x\|_{E}$, we have $\left\|x_{n}-x\right\|_{E} \rightarrow 0$. We say that the space $E$ has the Kadec-Klee property globally (resp. locally) in measure if each $x \in E$ is an $H_{g}$-point (resp. $H_{l}$-point) in $E$.

A point $x \geq 0$ is said to be an $H_{g}^{+}$-point of $E\left(H_{l}^{+}\right.$-point of $\left.E\right)$ if for any sequence $\left(x_{n}\right) \subset E_{+}$such that $x_{n} \rightarrow x$ globally (resp. locally) in measure and $\left\|x_{n}\right\|_{E} \rightarrow\|x\|_{E}$, we have $\left\|x_{n}-x\right\|_{E} \rightarrow 0$.

Let $0<p<\infty$. A quasi-Banach lattice $E$ is said to be $p$-convex whenever there is a constant $C>0$ such that

$$
\left\|\left(\sum_{i=1}^{n}\left|x_{i}\right|^{p}\right)^{1 / p}\right\|_{E} \leq C\left(\sum_{i=1}^{n}\left\|x_{i}\right\|_{E}^{p}\right)^{1 / p}
$$


for all $x_{1}, \ldots, x_{n} \in E, n \in N$. If $C=1$, we say that $E$ is $p$-convex with the constant 1. It is known that if $E$ is $p$-convex then there is an equivalent norm $\|\cdot\|_{1} \sim\|\cdot\|_{E}$ such that $\left(E,\|\cdot\|_{1}\right)$ is $p$-convex with the constant 1 . Moreover, $E$ is $p$-convex if and only if $E^{(1 / p)}$ is 1-convex (with the same constant). Finally, if $E$ is $p$-convex with the constant $C$ and $0<q<p$ then $E$ is $q$-convex with the constant at most $C$ (see Theorem 4.2 in [32]).

A quasi-Banach lattice $X$ is called $L$-convex whenever it is $p$-convex for some $p>0$ (see [16]).

\section{Quasi-Banach function spaces}

In this section we will prove some general results useful in the sequel which can be of independent interest. We will discuss also several basic results for quasi-Banach function spaces which in the case of Banach function spaces are well known (or even obvious). However, for a quasi-Banach function space the respective proofs need different techniques.

Lemma 3.1 Let $\left(E,\|\cdot\|_{E}\right)$ be a symmetric quasi-Banach function space. If $x \in E_{a}$ then $x^{*}(\infty)=0$.

Proof This implication is obvious under the assumption that $E \hookrightarrow L^{1}+L^{\infty}$ which is true for a symmetric Banach function space and need not be satisfied for arbitrary symmetric quasi-Banach function space. We will prove this fact in generality applying the condition (2.4). Suppose $a:=x^{*}(\infty)>0$. Denoting $A=\{t \in I:|x(t)| \geq a / 2\}$ we have $m(\mathrm{~A})=\infty$. Take a sequence $\left(A_{n}\right)$ of pairwise disjoint sets with $A_{n} \subset A$ and $m\left(A_{n}\right)=\infty$. Set $x_{n}=x \chi_{A_{n}}$. For each partition $x_{n}=y_{n}+z_{n}$ with $y_{n} \in L^{p, \infty}$ and $z_{n} \in L^{\infty}$ we have $\left\|z_{n}\right\|_{\infty} \geq a / 4$, because otherwise $y_{n}^{*}(\infty) \geq a / 4$ and consequently $y_{n} \notin L^{p, \infty}$. Thus $\left\|x_{n}\right\|_{L^{p, \infty}+L^{\infty}} \geq a / 4$, whence, by condition (2.4), we get $\left\|x_{n}\right\|_{E} \geq$ $a / 4 C_{2}$, where is $C_{2}$ is the constant from condition (2.4). This means that $x \notin E_{a}$.

Lemma 3.2 Let $\left(E,\|\cdot\|_{E}\right)$ be an L-convex quasi-Banach function space. If $\left\|x_{n}\right\|_{E} \rightarrow$ 0 then there is a subsequence $\left(x_{n_{m}}\right)_{m}$ of $\left(x_{n}\right)_{n}$, an element $x \in E_{+}$and a sequence $0 \leq \beta_{m} \rightarrow 0$ such that $\left|x_{n_{m}}\right| \leq \beta_{m} x$ for each $m$.

Proof Clearly, if $E$ is a Banach function space the result follows from [18, IV.2, Lemma 2 , p. 138]. Since $E$ is $L$-convex so it is $p$-convex for some $p>0$. Consequently $F=E^{(1 / p)}$ is 1-convex, it means there exists a norm $\|\cdot\|_{0}$ on $F$ which is equivalent to the quasi-norm $\|\cdot\|_{0} \sim\|\cdot\|_{F}$, where $\|z\|_{F}=\left(\left\||z|^{1 / p}\right\|_{E}\right)^{p}$. Suppose $\left\|x_{n}\right\|_{E} \rightarrow 0$ and set $y_{n}=\left|x_{n}\right|^{p}$. Then $y_{n} \in E^{(1 / p)}$ and $\left\|y_{n}\right\|_{F}=\left(\left\|\left|y_{n}\right|^{1 / p}\right\|_{E}\right)^{p} \rightarrow 0$. Consequently, $\left\|y_{n}\right\|_{0} \rightarrow 0$, whence there is a subsequence $\left(y_{n_{m}}\right)_{m}$ of $\left(y_{n}\right)_{n}$, an element $y \in F_{+}$and a sequence $0 \leq \alpha_{m} \rightarrow 0$ such that $\left|x_{n_{m}}\right|^{p}=\left|y_{n_{m}}\right| \leq \alpha_{m} y$ for each $m$ (see [18, IV.2, Lemma 2, p. 138]). Thus $\left|x_{n_{m}}\right|=\left|y_{n_{m}}\right|^{1 / p} \leq \alpha_{m}^{1 / p} y^{1 / p} \in E$. We finish the proof by taking $x=y^{1 / p}$ and $\beta_{m}=\alpha_{m}^{1 / p}$.

Remark 3.3 Clearly, if $\|\cdot\|_{E}$ is a norm then the implication $\left\|x_{n}-x\right\|_{E} \rightarrow 0 \Rightarrow$ $\left\|x_{n}\right\|_{E} \rightarrow\|x\|_{E}$ is trivial by the triangle inequality. If $\|\cdot\|_{E}$ is a quasi-norm then this is no longer true. Consider the space $R^{2}$ with the functional 


$$
\|x\|=\left\{\begin{array}{cl}
\frac{1}{2}\left|x_{1}\right| & \text { if } x_{2}=0 \\
\left|x_{1}\right|+\left|x_{2}\right| & \text { if } x_{2} \neq 0
\end{array}\right.
$$

for $x=\left(x_{1}, x_{2}\right)$. Clearly, $\|x+y\| \leq 2(\|x\|+\|y\|)$. Note also that for $u, v \in$ $\left(R^{2},\|\cdot\|\right)$ with $|u| \leq|v|$ we have $\|u\| \leq\|v\|$. Moreover, taking $x_{n}=(1,1 / n)$ and $x=(1,0)$ we get $\left\|x_{n}-x\right\|=\|(0,1 / n)\|=1 / n \rightarrow 0$, but $\left\|x_{n}\right\|=1+1 / n \nrightarrow$ $\|x\|=1 / 2$.

A necessary and sufficient condition for the implication $\left\|x_{n}-x\right\|_{E} \rightarrow 0 \Rightarrow$ $\left\|x_{n}\right\|_{E} \rightarrow\|x\|_{E}$, where $E$ is a quasi-Banach space, has been proved by Aoki (see Theorem 3 in [33]). We will show some useful sufficient condition for the above implication, a condition which is often applied in the studies of $E^{(*)}$. Namely, in the following Lemma we assume that $E$ is a quasi-Banach function space which is $p$-convex with the constant 1 for some $p>0$. Note that in the classical examples of symmetrization $E^{(*)}$ (the Lorentz space $E^{(*)}=\Lambda_{p, w}, E^{(*)}=\Lambda_{\phi}$ with $\phi\left(0^{+}\right)=0$, the Marcinkiewicz space $E^{(*)}=M_{\phi}^{(*)}$-see Sects. 4 and 6 for definitions, the generalized Orlicz-Lorentz space which is a symmetrization of the Musielak-Orlicz space-see $[12,13])$ the respective space $E$ satisfies trivially this condition.

Lemma 3.4 Suppose E is a quasi-Banach function space which is p-convex for some $p>0$ with the constant 1 . If $\left\|x_{n}-x\right\|_{E} \rightarrow 0$ then $\left\|x_{n}\right\|_{E} \rightarrow\|x\|_{E}$.

Proof If $p \geq 1$ and $E$ is $p$-convex with the constant 1 , then $E$ is 1 -convex with the constant 1 (see Theorem 4.2 in [32]), whence $\|\cdot\|_{E}$ is a norm. Consequently, we may assume that $0<p<1$. By the assumption $F=E^{(1 / p)}$ is 1 -convex with constant 1 , that is the functional $\|z\|_{F}=\left(\left\||z|^{1 / p}\right\|_{E}\right)^{p}$ is a norm. Suppose $\left\|x_{n}-x\right\|_{E} \rightarrow 0$ and set $y_{n}=\left|x_{n}\right|^{p}, y=|x|^{p}$. Then $y_{n}, y \in E^{(1 / p)}$. Since $0<p<1$ then the function $\varphi(u)=u^{1 / p}, u \geq 0$ is a convex function whence it is superadditive on $R_{+}$, that is $\varphi(|u-v|) \leq|\varphi(u)-\varphi(v)|, u, v \in R_{+}$. Consequently, by $y_{n}, y \geq 0$,

$$
\begin{aligned}
\left\|y_{n}-y\right\|_{F} & =\left(\left\|\left|y_{n}-y\right|^{1 / p}\right\|_{E}\right)^{p} \leq\left(\left\|y_{n}^{1 / p}-y^{1 / p}\right\|_{E}\right)^{p} \\
& =\left(\left\|\left|x_{n}\right|-|x|\right\|_{E}\right)^{p} \leq\left(\left\|\left|x_{n}-x\right|\right\|_{E}\right)^{p} \rightarrow 0 .
\end{aligned}
$$

By the triangle inequality, $\left\|y_{n}\right\|_{F} \rightarrow\|y\|_{F}$. Thus

$$
\left\|x_{n}\right\|_{E}^{p}=\left\|\left|x_{n}\right|\right\|_{E}^{p}=\left(\left\|\left|y_{n}\right|^{1 / p}\right\|_{E}\right)^{p} \rightarrow\left(\left\||y|^{1 / p}\right\|_{E}\right)^{p}=\|x\|_{E}^{p},
$$

which finishes the proof.

Lemma 3.5 If $x_{n} \rightarrow x$ globally in measure then $x_{n}^{*} \rightarrow x^{*}$ globally in measure. In particular, $x_{n}^{*}(\infty) \rightarrow x^{*}(\infty)$.

Proof This fact has been shown in the proof of Theorem 3.5 in [8] under assumption that $x^{*}(\infty)=0$. Let $a=x^{*}(\infty)>0$. By the assumption, $x_{n}^{*} \rightarrow x^{*}$ a.e. (see property $11^{\circ}$ in [29], page 67). First we claim that

$$
x_{n}^{*}(\infty) \rightarrow x^{*}(\infty)
$$


Suppose this is not the case. Suppose $b:=\lim \sup x_{n}^{*}(\infty)>a$. Passing to a subsequence if necessary we conclude that there is $N_{0}$ such that $x_{n}^{*}(\infty)>\frac{3}{4} b+\frac{1}{4} a$ for $n>N_{0}$. Moreover, there is $t_{0} \in I$ such that $x^{*}(t)<\frac{a+b}{2}$ for $t \geq t_{0}$. Then $x_{n}^{*}(t)-x^{*}(t) \geq \frac{b-a}{4}$ for $t \geq t_{0}$ and $n>N_{0}$. This is a contradiction with the fact that $x_{n}^{*} \rightarrow x^{*}$ a.e.

If $c:=\liminf x_{n}^{*}(\infty)<a$ then there is $N_{0}$ such that $x_{n}^{*}(\infty)<\frac{a+c}{2}$ for $n \geq N_{0}$ (passing to a subsequence if necessary). Consequently,

$$
m\left\{t \in I: x_{n}(t)>\frac{a+c}{2}\right\}=m\left\{t \in I: x_{n}^{*}(t)>\frac{a+c}{2}\right\}<\infty \text { for } n \geq N_{0} .
$$

Moreover, $m\left\{t \in I: x(t)>\frac{3 a+c}{4}\right\}=m\left\{t \in I: x^{*}(t)>\frac{3 a+c}{4}\right\}=\infty$, whence

$$
m\left\{t \in I:\left|x_{n}(t)-x(t)\right|>\frac{a-c}{4}\right\}=\infty \text { for } n \geq N_{0} .
$$

Thus $x_{n} \nrightarrow x$ globally in measure, which proves the claim (3.1).

Let $\varepsilon>0$. There exists $t_{\varepsilon}>0$ and $N_{0} \in N$ such that

$$
x^{*}(t)<x^{*}(\infty)+\varepsilon / 4 \text { for all } t \geq t_{\varepsilon} \text { and }\left|x_{n}^{*}\left(t_{\varepsilon}\right)-x^{*}\left(t_{\varepsilon}\right)\right|<\varepsilon / 4 \text { for } n \geq N_{0} .
$$

Furthermore, by (3.1), there is $N_{1}$ such that

$$
\left|x_{n}^{*}(\infty)-x^{*}(\infty)\right|<\varepsilon / 4 \text { for } n \geq N_{1} .
$$

Since $x_{n}^{*}$, for $n \in N$, and $x^{*}$ are nonincreasing functions,

$$
m\left(\left\{t \in\left[t_{\varepsilon}, \infty\right):\left|x_{n}^{*}(t)-x^{*}(t)\right|>\varepsilon\right\}\right)=0 \text { for } n \geq \max \left\{N_{0}, N_{1}\right\} .
$$

Since $x_{n}^{*} \rightarrow x^{*}$ a.e., $x_{n}^{*}$ converges to $x^{*}$ locally in measure. Thus

$$
m\left(\left\{t \in\left(0, t_{\varepsilon}\right]:\left|x_{n}^{*}(t)-x^{*}(t)\right|>\varepsilon\right\}\right) \rightarrow 0 .
$$

Hence $x_{n}^{*}$ converges to $x^{*}$ in measure.

Proposition 3.6 Suppose $E$ is a quasi-Banach function space. If $x$ is an $H_{l}$-point ( $\mathrm{H}_{l}^{+}$-point) of $E$ then $x$ is a point of order continuity.

Proof For a Banach function space the result follows from Proposition 2.1 in [11] (see also [6, Proposition 1.1]). If $x \notin E_{a}$, by Theorem 2.1, there is a sequence of pairwise disjoint elements $\left(x_{n}\right)_{n=1}^{\infty}$ with $0 \leq x_{n} \leq|x|$ and $\left\|x_{n}\right\| \geq \delta>0$. Taking $z_{n}=|x|-x_{n}$ we can finish the proof as in [11, Proposition 2.1].

Lemma 3.7 Let $E$ be a quasi-Banach function space. If $x \geq 0$ is an $H_{g}^{+}$-point of $E$, then $\left\|x \chi_{A_{k}}\right\|_{E} \rightarrow 0$, where $A_{k}=\{t \in I: x(t)<1 / k\}$. Moreover, if $x(x \geq 0)$ is an $H_{g}$-point of $E\left(H_{g}^{+}\right.$-point of $\left.E\right)$, then $\left\|x \chi_{B_{n}}\right\|_{E} \rightarrow 0$ for each sequence $\left(B_{n}\right)$ of measurable sets satisfying $m\left(B_{n}\right) \rightarrow 0$. 
Proof See the proof of Remark 3.4 in [23]. For the second claim see Lemma 3.2 in [8], still true for a quasi-norm (the symmetry of $E$ is not necessary in the proof).

Remark 3.8 Clearly, the above Lemma is also true for an $H_{l}^{+}$-point ( $H_{l}$-point).

Theorem 3.9 Let $E$ be an L-convex quasi-Banach function space and $x \in E$. Then the following statements are equivalent:

(i) $x$ is an $H_{g}$-point of $E$.

(ii) $|x|$ is an $H_{g}$-point of $E$.

(iii) $|x|$ is an $H_{g}^{+}$-point of $E$.

Remark 3.10 For an $H_{l}$-point one can prove similarly the analogous result.

Proof of Theorem The implication (i) $\Rightarrow$ (ii) follows the same way as in the proof of Lemma 3.1 in [8] (also for a quasi-normed space, the assumption that $E$ is $L$-convex is not needed in this part). The implication (ii) $\Rightarrow$ (iii) is obvious.

The implication (iii) $\Rightarrow$ (i). We apply the proof of Lemma 3.5 from [23]. However, we need to modify it essentially, so we present the details for reader's convenience. Let $\left\|x_{n}\right\| \rightarrow\|x\|$ and $x_{n} \rightarrow x$ in measure. Since for each $\eta>0$ we have

$$
m\left\{t \in I:|| x_{n}(t)|-| x(t) \| \geq \eta\right\} \leq m\left\{t \in I:\left|x_{n}(t)-x(t)\right| \geq \eta\right\} \rightarrow 0,
$$

so $\left|x_{n}\right| \rightarrow|x|$ in measure. By the assumption we conclude that

$$
\left\|\left|x_{n}\right|-|x|\right\| \rightarrow 0 \text {. }
$$

Applying Lemma 3.2, we find a subsequence $\left(\left|x_{n_{m}}\right|\right)_{m}$ of $\left(\left|x_{n}\right|\right)_{n}$, an element $y \in E_{+}$ and a sequence $0 \leq \beta_{m} \rightarrow 0$ such that ||$x_{n_{m}}|-| x|| \leq \beta_{m} y$ for each $m$. Let $\varepsilon>0$. Fix $m_{0}$ satisfying

$$
\left\|\beta_{m_{0}} y\right\|<\varepsilon / 16 C^{4}
$$

where $C=C_{E}$ is from the $C$-triangle inequality for $\|\cdot\|$. Denote $\left(\left|x_{n_{m}}\right|\right)_{m=m_{0}}^{\infty}$ still by $\left(\left|x_{n}\right|\right)_{n=1}^{\infty}$ and $\left(\beta_{m}\right)_{m=m_{0}}^{\infty}$ by $\left(\beta_{n}\right)_{n=1}^{\infty}$. We may assume that $\beta_{n}$ is decreasing, whence

$$
|| x_{n}|-| x \| \leq \beta_{n} y \leq \beta_{1} y \text { and }\left\|\beta_{1} y\right\|<\varepsilon / 16 C^{4}
$$

for all $n=1,2$. Set $u=|x|+\beta_{1} y$ and

$$
B_{k}=\{t \in I: u(t)<1 / k\} .
$$

Since $B_{k} \subset\{t \in I:|x(t)|<1 / k\}$, by Lemma 3.7, we take $k$ big enough to satisfy

$$
\left\|x \chi_{B_{k}}\right\|<\varepsilon / 16 C^{3} \text {. }
$$

Applying the second inequality from (3.3) we conclude that

$$
\left\|u \chi_{B_{k}}\right\| \leq C\left(\left\|x \chi_{B_{k}}\right\|+\left\|\beta_{1} y \chi_{B_{k}}\right\|\right)<\varepsilon / 8 C^{2} .
$$


Moreover, $\left\|\left|x_{n}\right|-|x|\right\|<\varepsilon / 8 C^{3}$ for sufficiently large $n$. Setting

$C_{n}=\left\{t \in I: 0 \leq \operatorname{sgn}\left[x(t) x_{n}(t)\right] \leq 1\right\}$ and $D_{n}=\left\{t \in I: \operatorname{sgn}\left[x(t) x_{n}(t)\right]=-1\right\}$, we get

$\left\|x-x_{n}\right\| \leq C\left\|\left(|x|-\left|x_{n}\right|\right) \chi_{C_{n}}\right\|+C^{2}\left\|\left(x-x_{n}\right) \chi_{D_{n} \cap B_{k}}\right\|+C^{2}\left\|\left(x-x_{n}\right) \chi_{D_{n} \backslash B_{k}}\right\|$.

Note that

$$
\begin{aligned}
C^{2}\left\|\left(x-x_{n}\right) \chi_{D_{n} \cap B_{k}}\right\| & =C^{2}\left\|\left(|x|+\left|x_{n}\right|\right) \chi_{D_{n} \cap B_{k}}\right\| \\
& \leq C^{3}\left(\left\|\left(\left|x_{n}\right|-|x|\right) \chi_{D_{n} \cap B_{k}}\right\|+\left\|2|x| \chi_{D_{n} \cap B_{k}}\right\|\right) \\
& \leq C^{3}\left(\varepsilon / 8 C^{3}+\varepsilon / 8 C^{3}\right) .
\end{aligned}
$$

Consequently,

$$
\left\|x-x_{n}\right\| \leq 3 \varepsilon / 8+C^{2}\left\|\left(x-x_{n}\right) \chi_{D_{n} \backslash B_{k}}\right\| .
$$

Take

$$
0<\delta<\frac{\varepsilon}{16 k C^{4}} \min \left\{\frac{1}{\left\|\beta_{1} y\right\|}, \frac{1}{\|x\|}\right\} .
$$

Denote

$$
\begin{aligned}
& F_{n}^{k}=\left\{t \in D_{n} \backslash B_{k}:\left|x_{n}(t)-x(t)\right|>\delta\right\}, \\
& G_{n}^{k}=\left\{t \in D_{n} \backslash B_{k}:\left|x_{n}(t)-x(t)\right| \leq \delta\right\} .
\end{aligned}
$$

Note that $x_{n} \stackrel{m}{\rightarrow} x$ and consequently $m\left(F_{n}^{k}\right) \rightarrow 0$ as $n \rightarrow \infty$. Since $|x|$ is $H_{g}^{+}$-point, by Lemma 3.7, we get $\left\||x| \chi_{F_{n}^{k}}\right\|<\varepsilon / 32 C^{4}$ for sufficiently large $n$. Thus, by (3.3),

$$
\begin{aligned}
\left\|\left(x-x_{n}\right) \chi_{D_{n} \backslash B_{k}}\right\| \leq & C\left\|\left(x-x_{n}\right) \chi_{F_{n}^{k}}\right\|+C\left\|\left(x-x_{n}\right) \chi_{G_{n}^{k}}\right\| \\
\leq & C\left\|\left(2|x|+\beta_{1} y\right) \chi_{F_{n}^{k}}\right\|+C\left\|\left(x-x_{n}\right) \chi_{G_{n}^{k}}\right\| \\
< & C^{2}\left(2\left\||x| \chi_{F_{n}^{k}}\right\|+\left\|\beta_{1} y \chi_{F_{n}^{k}}\right\|\right) \\
& +C\left\|\left(x-x_{n}\right) \chi_{G_{n}^{k}}\right\|<\varepsilon / 8 C^{2}+C\left\|\left(x-x_{n}\right) \chi_{G_{n}^{k}}\right\|
\end{aligned}
$$

for sufficiently large $n$. Finally, divide set $G_{n}^{k}$ in two subsets

$$
H_{n}^{k}=\left\{t \in G_{n}^{k}:|x(t)| \geq \frac{1}{2 k}\right\} \text { and } I_{n}^{k}=\left\{t \in G_{n}^{k}: \beta_{1} y(t) \geq \frac{1}{2 k}\right\}
$$


Note that $G_{n}^{k} \subset H_{n}^{k} \cup I_{n}^{k}$, because if $t \notin H_{n}^{k} \cup I_{n}^{k}$ then $u(t)<1 / k$ so $t \in B_{k}$ and $t \notin G_{n}^{k}$. Therefore, by (3.5),

$$
\begin{aligned}
\left\|\left(x-x_{n}\right) \chi_{G_{n}^{k}}\right\| & \leq \delta C\left(\left\|\chi_{H_{n}^{k}}\right\|+\left\|\chi_{I_{n}^{k}}\right\|\right) \leq \delta C\left(\left\|2 k|x| \chi_{H_{n}^{k}}\right\|+\left\|2 k \beta_{1} y \chi_{I_{n}^{k}}\right\|\right) \\
& \leq 2 k \delta C\left(\left\||x| \chi_{H_{n}^{k}}\right\|+\left\|\beta_{1} y \chi_{I_{n}^{k}}\right\|\right)<\varepsilon / 4 C^{3} .
\end{aligned}
$$

Combining (3.4), (3.6) and (3.7) we get

$$
\begin{aligned}
\left\|x-x_{n}\right\| & \leq 3 \varepsilon / 8+C^{2}\left\|\left(x-x_{n}\right) \chi_{D_{n} \backslash B_{k}}\right\| \\
& \leq 3 \varepsilon / 8+C^{2}\left(\varepsilon / 8 C^{2}+C\left\|\left(x-x_{n}\right) \chi_{G_{n}^{k}}\right\|\right) \\
& \leq \varepsilon / 2+C^{3} \varepsilon / 4 C^{3} \leq \varepsilon
\end{aligned}
$$

for sufficiently large $n$.

Lemma 3.11 Suppose $E$ is a symmetric quasi-Banach function space which is $p$ convex with the constant 1 for some $0<p<1$. Let $F=E^{(1 / p)}$. If $x$ is an $H_{g}$-point of $E$ then $x^{p}$ is an $H_{g}$-point of $F$.

Proof By Theorem 3.9 it is enough to show that $|x|^{p}$ is an $H_{g}^{+}$-point of $F$. Assume that $0 \leq x_{n} \rightarrow|x|^{p}$ globally in measure and $\left\|x_{n}\right\|_{F} \rightarrow\left\||x|^{p}\right\|_{F}$. Thus $x_{n}^{1 / p} \rightarrow|x|$ globally in measure by Lemma 3.9 (i) from [23] (note that $F$ is symmetric). Moreover,

$$
\left\|x_{n}^{1 / p}\right\|_{E}^{p}=\left\|x_{n}\right\|_{F} \rightarrow\left\||x|^{p}\right\|_{F}=\||x|\|_{E}^{p}
$$

whence $\left\|x_{n}^{1 / p}\right\|_{E} \rightarrow\||x|\|_{E}$. Since $|x|$ is an $H_{g}$-point of $E$ (see Theorem 3.9), so $\left\|x_{n}^{1 / p}-|x|\right\|_{E} \rightarrow 0$. Consequently,

$$
\left\|x_{n}-|x|^{p}\right\|_{F}=\left\|\left.\left.\left|x_{n}-\right| x\right|^{p}\right|^{1 / p}\right\|_{E}^{p} \leq\left\|\left|x_{n}^{1 / p}-\right| x||\right\|_{E}^{p} \rightarrow 0
$$

because $\varphi(u)=u^{1 / p}, u \geq 0$, is a convex function whence it is superadditive on $R_{+}$.

The following Proposition has been proved in [8, Lemma 3.8] for a symmetric Banach function space.

Proposition 3.12 Suppose $E$ is a symmetric quasi-Banach function space which is $p$-convex with the constant 1 for some $p \in(0,1)$. If $x$ is an $H_{g}$-point of $E$ and $x_{n} \rightarrow x$ globally in measure then $\|x\|_{E} \leq \liminf \left\|x_{n}\right\|_{E}$.

Proof Suppose $x_{n} \rightarrow x$ globally in measure and $x_{n}, x \in E$. Let $F=E^{(1 / p)}$. Set $y_{n}=\left|x_{n}\right|^{p}, y=|x|^{p}$. Then $y, y_{n} \in F$. Moreover, $\left|x_{n}\right| \rightarrow|x|$ in measure, whence $y_{n} \rightarrow y$ globally in measure, by Lemma 3.9 (ii) from [23] with $\varphi(u)=u^{1 / p}$. By 
Lemma 3.11, $y$ is an $H_{g}$-point of $F$. We assume in the paper that $E \in(F P)$, where $F P$ denotes the Fatou property defined in Sect. 2. Consequently, $F \in(F P)$. Since $F$ is a symmetric Banach function space, by Lemma 3.8 in [8], we conclude that

$$
\||x|\|_{E}^{p}=\|y\|_{F} \leq \liminf \left\|y_{n}\right\|_{F}=\liminf \left\|\left|x_{n}\right|\right\|_{E}^{p} .
$$

Lemma 3.13 Let $E$ be a symmetric quasi-Banach function space.

(i) If $x^{*}(\infty)=0$ and $x \in E$ is an $H_{g}$-point in $E$, then $x^{*}$ is an $H_{g}$-point in $E$.

(ii) If $x \in E$ is an $H_{l}$-point in $E$, then $x^{*}$ is an $H_{l}$-point in $E$.

Proof (i) We follow as in the proof of Theorem 3.3 in [8], applying additionally Theorem 3.9, the implication (i) $\Rightarrow$ (ii), which is true without the assumption that $E$ is $L$-convex. (ii) If $x$ is an $H_{l}$-point, by Proposition 3.6 and Lemma 3.1, we have $x^{*}(\infty)=0$. Then the proof is analogous as in (i).

Lemma 3.14 Let $E$ be a symmetric quasi-Banach function space on I. If $x \in E$ is an $H_{g}$-point, then for each sequence $\left(d_{n}\right)$ in $L^{0}$ with $0 \leq d_{n} \leq|x|$ and $d_{n} \rightarrow 0$ globally in measure we have $\left\|d_{n}\right\|_{E} \rightarrow 0$.

Proof We follow as in the proof of Lemma 3.7 in [8], applying additionally Lemmas 3.13 and 3.7 .

Theorem 3.15 Let $E$ be a quasi-Banach function space and $0 \leq x \in E$. Consider the following statements:

(1) The point $x$ is an $H_{g}^{+}$-point of $E$.

(2) (i) for each sequence $\left(d_{n}\right) \subset E_{+}$such that $d_{n} \leq x$ and $d_{n} \rightarrow 0$ globally in measure we have $\left\|d_{n}\right\|_{E} \rightarrow 0$.

(ii) for each sequence $\left(y_{n}\right) \subset E_{+}$such that $x \leq y_{n}, y_{n} \rightarrow x$ globally in measure and $\left\|y_{n}\right\|_{E} \rightarrow\|x\|_{E}$, we have $\left\|y_{n}-x\right\|_{E} \rightarrow 0$.

The implication $(1) \Rightarrow(2)$ is satisfied provided $E$ is symmetric. If $E$ is p-convex with the constant 1 for some $p>0$, then the implication (2) $\Rightarrow$ (1) holds.

Proof The implication (1) $\Rightarrow$ (2) is true by Lemma 3.14.

The implication (2) $\Rightarrow$ (1). Suppose $0 \leq x_{n} \rightarrow x$ globally in measure, $\left\|x_{n}\right\|_{E} \rightarrow$ $\|x\|_{E}$ and $x_{n}, x \in E$. Set

$$
A_{n}=\left\{t \in I: x(t) \geq x_{n}(t)\right\}
$$

Then $0 \leq\left(x-x_{n}\right) \chi_{A_{n}} \leq x$. By (i), we have $\left\|\left(x-x_{n}\right) \chi_{A_{n}}\right\|_{E} \rightarrow 0$. Clearly, we may assume that $0<p \leq 1$, since otherwise $E$ is just 1-convex with the constant 1 (by Theorem 4.2 in [32]), whence it is a normed space and the proof below is simpler. We have

$$
\begin{aligned}
x_{n} & \leq \max \left\{x_{n}, x\right\} \leq x_{n}+\left(x-x_{n}\right) \chi_{A_{n}}=\left[\left(x_{n}+\left(x-x_{n}\right) \chi_{A_{n}}\right)^{p}\right]^{1 / p} \\
& \leq\left[x_{n}^{p}+\left(\left(x-x_{n}\right) \chi_{A_{n}}\right)^{p}\right]^{1 / p},
\end{aligned}
$$


because the function $\varphi(u)=u^{p}$ for $0<p \leq 1$ is subadditive on $R_{+}$. Taking into account that $E$ is $p$-convex with the constant 1 we get

$$
\begin{aligned}
& \left\|x_{n}\right\|_{E} \leq\left\|\max \left\{x_{n}, x\right\}\right\|_{E} \leq\left\|\left[x_{n}^{p}+\left(\left(x-x_{n}\right) \chi_{A_{n}}\right)^{p}\right]^{1 / p}\right\|_{E} \\
& \quad \leq\left(\left\|x_{n}\right\|_{E}^{p}+\left\|\left(x-x_{n}\right) \chi_{A_{n}}\right\|_{E}^{p}\right)^{1 / p}
\end{aligned}
$$

whence $\left\|\max \left\{x_{n}, x\right\}\right\|_{E} \rightarrow\|x\|_{E}$. Since $\max \left\{x_{n}, x\right\} \rightarrow x$ globally in measure, by (ii),

$$
\left\|\left(x_{n}-x\right) \chi_{I \backslash A_{n}}\right\|_{E}=\left\|\max \left\{x_{n}, x\right\}-x\right\|_{E} \rightarrow 0 .
$$

Thus $\left\|x-x_{n}\right\|_{E} \rightarrow 0$.

Theorem 3.16 Let $E$ be a quasi-Banach function space and $0 \leq x \in E$. Consider the following statements:

(1) The point $x$ is an $\mathrm{H}_{l}^{+}$-point of $E$.

(2) (i) for each sequence $\left(d_{n}\right) \subset E_{+}$such that $d_{n} \leq x$ and $d_{n} \rightarrow 0$ locally in measure we have $\left\|d_{n}\right\|_{E} \rightarrow 0$.

(ii) for each sequence $\left(y_{n}\right) \subset E_{+}$such that $x \leq y_{n}, y_{n} \rightarrow x$ locally in measure and $\left\|y_{n}\right\|_{E} \rightarrow\|x\|_{E}$, we have $\left\|y_{n}-x\right\|_{E} \rightarrow 0$.

Then the implication (1) $\Rightarrow$ (2) is true. If $E$ is $p$-convex with the constant 1 then the implication (2) $\Rightarrow$ (1) holds.

Proof The implication (1) $\Rightarrow$ (2) (i) follows from Proposition 3.6 and Theorem 2.1. The proof of implication $(2) \Rightarrow$ (1) follows as for $\mathrm{H}_{g}^{+}$-point in the above theorem.

\section{Kadec-Klee properties in symmetrizations}

For a quasi-Banach function space $E$ on $I=(0,1)$ or $I=(0, \infty)$ define a space $E^{(*)}$ (symmetrization of $E$ ) as

$$
E^{(*)}=\left\{x \in L^{0}(I): x^{*} \in E\right\},
$$

with the functional $\|x\|_{E^{(*)}}=\left\|x^{*}\right\|_{E}$.

The dilation operator $D_{s}, s>0$, is defined by $D_{s} x(t)=x(t / s), t \in I$ if $I=$ $(0, \infty)$ and

$$
D_{s} x(t)=\left\{\begin{array}{cl}
x(t / s) & \text { if } t<\min \{1, s\} \\
0 & \text { if } \quad s \leq t<1
\end{array}\right.
$$

for $t \in I=(0,1)$. The operator $D_{s}$ is bounded in any symmetric space $E$ on $I$ and $\left\|D_{S}\right\|_{E \rightarrow E} \leq \max (1, s)$ (see [34, Lemma 1] in the case $I=(0,1),[29$, pp. 96-98] for $I=(0, \infty)$ and [31, p. 130] for both cases). A. Kamińska and Y. Raynaud showed that $\|\cdot\|_{E^{(*)}}$ is a quasi-norm if and only if there is a constant $K>0$ such that

$$
\left\|D_{2} x^{*}\right\|_{E} \leq K\left\|x^{*}\right\|_{E} \text { for all } x^{*} \in E
$$


(see Lemma 1.4 in [22]). In this case the quasi-norm constant $C_{E^{(*)}}$ is equal to $K C_{E}$, where $C_{E}$ is the respective quasi-norm constant for the space $E$ (see [22]). Therefore we always assume in this section that the condition (4.1) is satisfied.

Let $\phi: I \rightarrow(0, \infty)$ be a quasi-concave function, that is, $\phi$ is positive, nondecreasing and $\phi(t) / t$ is nonincreasing for $t \in(0, m(I))$. Then the Marcinkiewicz space $M_{\phi}^{(*)}$ is defined as

$$
M_{\phi}^{(*)}=M_{\phi}^{(*)}(I)=\left\{x \in L^{0}(I):\|x\|_{M_{\phi}^{(*)}}=\sup _{t \in I} \phi(t) x^{*}(t)<\infty\right\} .
$$

Moreover, if $\phi: I \rightarrow(0, \infty)$ is a concave function, $\phi$ is positive and nondecreasing, then the Lorentz function space $\Lambda_{\phi}$ is given by the norm

$$
\|x\|_{\Lambda_{\phi}}=\int_{I} x^{*}(t) d \phi(t)=\phi\left(0^{+}\right)\|x\|_{L^{\infty}(I)}+\int_{I} x^{*}(t) \phi^{\prime}(t) d t .
$$

The Marcinkiewicz spaces $M_{\phi}^{(*)}$ and Lorentz spaces $\Lambda_{\phi}$ are symmetric quasi-Banach function spaces, symmetric Banach function spaces on $I$, respectively (see $[3,29])$. Clearly, $M_{\phi}^{(*)}=\left(L^{\infty}(\phi)\right)^{(*)}$. Moreover, $\Lambda_{\phi}=\left(L^{1}\left(\phi^{\prime}\right)\right)^{(*)}$ provided $\phi\left(0^{+}\right)=0$ (see [25] for some properties and more references). Clearly, $L^{\infty}(\phi), L^{1}\left(\phi^{\prime}\right)$ are the respective weighted spaces according to the definition (2.2), for example $\|x\|_{L^{1}\left(\phi^{\prime}\right)}=$ $\int_{I}|x(t)| \phi^{\prime}(t) d t$.

The spaces $E^{(*)}$ have been studied among others in the papers [12,13,21,22,2426]. Kamińska and Raynaud studied the relationships between the structure of $E^{(*)}$ and the structure of $E$ (see [22]). The nature of our consideration is analogous.

Let $P$ be a local property of a point $x \in E$ (an $H_{g}$-point, an $H_{l}$-point, etc.). We say that $x=x^{*}$ is a $P^{*}$-point provided it is a $P$-point but restricted to nonnegative and nonincreasing elements only.

For example, a point $x=x^{*}$ is said to be an $H_{g}^{*}$-point of $E\left(H_{l}^{*}\right.$-point of $E$ ) whenever for any sequence $\left(x_{n}\right) \subset E, x_{n}=x_{n}^{*}$ such that $x_{n} \rightarrow x$ globally (resp. locally) in measure and $\left\|x_{n}\right\|_{E} \rightarrow\|x\|_{E}$, we have $\left\|x_{n}-x\right\|_{E} \rightarrow 0$.

Similarly, for a global property $G$, we say that $E$ has a property $G^{*}$ if $E$ satisfies the property $G$ but restricted to nonnegative and nonincreasing elements only.

Namely, a space $E$ is said to have $H_{g}^{*}\left(H_{l}^{*}\right)$ property provided each $x=x^{*} \in E$ is an $H_{g}^{*}$-point of $E\left(H_{l}^{*}\right.$-point of $\left.E\right)$.

Obviously, if $E \in(G)$, then $E \in\left(G^{*}\right)$. The natural question of the converse implication has been considered in [5] (for rotundity properties) and in [24,25] (for monotonicity properties and for an order continuity).

Lemma 4.1 Let $E$ be a quasi-Banach function space.

(i) Suppose $x=x^{*} \in E$ is an $H_{g}^{*}$-point. Then $\left\|x \chi_{A_{k}}\right\|_{E} \rightarrow 0$ as $k \rightarrow \infty$, where $A_{k}=\{t \in I: x(t)<1 / k\}$. In particular, if $x^{*}(\infty)=0$ then $\left\|x \chi_{(n, \infty)}\right\|_{E} \rightarrow 0$ as $n \rightarrow \infty$.

Proof See the proof of Remark 3.4 in [23]. 
The following remark will be important for the main results in this section.

Remark 4.2 Let $E$ be a quasi-Banach function space and $x=x^{*} \in E$. Each of the following implications is not true in general:

(i) $x$ is an $H_{g}^{*}$-point of $E \Rightarrow x$ is an $H_{g}$-point of $E$.

(ii) $x$ is an $H_{g}^{*}$-point of $E \Rightarrow\left\|x \chi_{\left(0, a_{n}\right)}\right\|_{E} \rightarrow 0$ for any sequence $a_{n} \rightarrow 0$.

(iii) $x$ is an $H_{g}^{*}$-point of $E \Rightarrow x$ is an $H_{g}$-point of $E^{(*)}$.

Consider $E=L^{\infty}(w)$ with $w(t)=1-t$ on $I=(0,1)$. Then $E$ has no $H_{g}$-points. Indeed, if $0 \neq x \in E$ then there is a number $\delta>0$ and a measurable set $A$ of positive measure such that $|x(t)| \geq \delta$ for $t \in A$. Moreover, we can assume that $A \subset\left[\lambda_{1}, \lambda_{2}\right]$ with $\lambda_{1}<\lambda_{2}<1$. Take a sequence $\left(A_{n}\right)$ of measurable subsets of $A$ with $0<m\left(A_{n}\right) \rightarrow 0$. Then $\left\|x \chi_{A_{n}}\right\|_{E} \geq \delta\left(1-\lambda_{2}\right)>0$, whence $x$ is not an $H_{g}$-point by Lemma 3.7. Let

$$
x=\chi_{(0,1)} .
$$

Clearly, $\left\|x \chi_{(0,1 / n)}\right\|_{E} \nrightarrow 0$. We will show that $x$ is an $H_{g}^{*}$-point of $E$. Consider a sequence $\left(x_{n}\right) \subset E, x_{n}=x_{n}^{*}$ such that $x_{n} \rightarrow x$ globally in measure and $\left\|x_{n}\right\|_{E} \rightarrow$ $\|x\|_{E}$. Note that $\|x\|_{E}=1$. Moreover, $x_{n}\left(0^{+}\right) \rightarrow 1$ because otherwise $\left\|x_{n}\right\|_{E} \nrightarrow$ $\|x\|_{E}$. Let $\varepsilon>0$. Take a number $\eta$ satisfying $1-\varepsilon / 2<\eta<1$. Since $x_{n} \rightarrow x$ globally in measure and $x_{n}=x_{n}^{*}$, so $x_{n}(\eta) \rightarrow 1$. Thus $x_{n} \rightarrow x$ uniformly in $(0, \eta]$. Consequently, $\left\|\left(x_{n}-x\right) \chi_{(0, \eta]}\right\|_{L^{\infty}}<\varepsilon / 2$ for sufficiently large $n$ and

$\left\|x_{n}-x\right\|_{E} \leq\left\|\left(x_{n}-x\right) \chi_{(0, \eta]}\right\|_{E}+\left\|\left(x_{n}-x\right) \chi_{(\eta, 1)}\right\|_{E} \leq\left(w\left(0^{+}\right) \varepsilon / 2+1-\eta\right)<\varepsilon$

for sufficiently large $n$.

Note also that condition (4.1) is satisfied for the space $E$, because for each $x=$ $x^{*} \in E$ we have $\left\|D_{2} x\right\|_{E}=\|x\|_{E}$.

Finally, $x$ is not an $H_{g}$-point of $E^{(*)}$. Indeed, let $x_{n}=\chi_{(0,1 / 2]}+\chi_{(1 / 2+1 / n, 1)}$. Then $x_{n} \rightarrow x$ globally in measure, $\left\|x_{n}\right\|_{E^{(*)}}=\|x\|_{E^{(*)}}$. On the other hand, $\left\|x_{n}-x\right\|_{E^{(*)}}=$ 1 .

In view Remark 4.2, two assumptions in the below lemma are independent. Moreover, without the second assumption this lemma is not true in general.

Lemma 4.3 Let $E$ be a quasi-Banach function space on I (not necessary symmetric). If $x=x^{*} \in E$ is an $H_{g}^{*}$-point and $\left\|x \chi_{\left(0, a_{n}\right)}\right\|_{E} \rightarrow 0$ for any sequence $a_{n} \rightarrow 0$, then for each sequence $\left(d_{n}\right)$ in $L^{0}$ with $0 \leq d_{n}=d_{n}^{*} \leq x$ and $d_{n} \rightarrow 0$ globally in measure we have $\left\|d_{n}\right\|_{E} \rightarrow 0$.

Proof An analogous result for an $H_{g}$-point has been proved in [8, Lemma 3.7] under the assumption that $E$ is a symmetric Banach function space on $I$. Although the below proof is similar we present it for the reader's convenience (because of the essential change in the assumptions). Assume for the contrary that $0 \leq d_{n}=d_{n}^{*} \leq x, d_{n} \rightarrow 0$ globally in measure and $\left\|d_{n}\right\|_{E} \nrightarrow 0$. Passing to a subsequence if necessary, we have $\left\|d_{n}\right\|_{E} \geq \delta>0$ for some $\delta>0$. Setting $A_{n}(\varepsilon)=\left\{t \in I: d_{n}(t)>\varepsilon\right\}$, we have 
$m\left(A_{n}(\varepsilon)\right) \rightarrow 0$ for each $\varepsilon>0$, whence $A_{n}(\varepsilon)=\left(0, a_{n}(\varepsilon)\right)$ for some sequence $a_{n} \rightarrow 0$. By the assumption,

$$
\left\|d_{n} \chi_{A_{n}(\varepsilon)}\right\|_{E} \leq\left\|x \chi_{\left(0, a_{n}(\varepsilon)\right)}\right\|_{E} \rightarrow 0
$$

for each $\varepsilon>0$. Passing to a subsequence if necessary, we may assume that

$$
\left\|d_{n} \chi_{A_{n}\left(\varepsilon_{n}\right)}\right\|_{E}<1 / n \text { and } m\left(A_{n}\left(\varepsilon_{n}\right)\right)<1 / n,
$$

where $\varepsilon_{n}=1 / n$.

Suppose $x^{*}(\infty)>0$. Then $\left\|\chi_{I}\right\|_{E}<\infty$. Notice that $\left\|d_{n} \chi_{I \backslash A_{n}\left(\varepsilon_{n}\right)}\right\|_{E} \geq \delta / 2 C_{E}$ for sufficiently large $n \in N$. Therefore

$$
\delta / 2 C_{E} \leq\left\|d_{n} \chi_{I \backslash A_{n}\left(\varepsilon_{n}\right)}\right\|_{E} \leq \frac{1}{n}\left\|\chi_{I}\right\|_{E}
$$

for $n \in N$ large enough, a contradiction.

Consequently, $x^{*}(\infty)=0$. For each $\varepsilon_{n}$ there is $t_{\varepsilon_{n}}$ satisfying $x^{*}\left(t_{\varepsilon_{n}}\right) \leq \varepsilon_{n}$. Set

$$
t_{\varepsilon_{n}}=\inf \left\{t: x^{*}(t) \leq \varepsilon_{n}\right\}
$$

First we claim that

$$
\left\|\varepsilon_{n} \chi_{\left(0, t_{\varepsilon_{n}}\right)}\right\|_{E} \rightarrow 0 .
$$

Otherwise, set $z_{n}=\left(x^{*}-\varepsilon_{n}\right) \chi_{\left(0, t_{\varepsilon_{n}}\right)}$. Then $z_{n}$ is a nonincreasing function, that is $z_{n}^{*}=z_{n}$ for each $n$. Moreover, $\left|z_{n}-x^{*}\right|=\varepsilon_{n} \chi_{\left(0, t_{\varepsilon_{n}}\right)}+x^{*} \chi_{\left[t_{\varepsilon_{n}}, \infty\right)}$. Note that $t_{\varepsilon_{n}} \rightarrow$ $\infty$ when $m\left(\operatorname{supp} x^{*}\right)=\infty$ and $t_{\varepsilon_{n}} \rightarrow m\left(\operatorname{supp} x^{*}\right)$ if $m\left(\operatorname{supp} x^{*}\right)<\infty$. In both cases we conclude that $z_{n} \uparrow x^{*}$ globally in measure. Consequently, by $E \in(F P)$, $\left\|z_{n}\right\|_{E} \rightarrow\left\|x^{*}\right\|_{E}$. On the other hand,

$$
\left\|z_{n}-x^{*}\right\|_{E} \geq\left\|\varepsilon_{n} \chi_{\left(0, t_{\varepsilon_{n}}\right)}\right\|_{E} \nrightarrow 0,
$$

a contradiction with $x^{*}$ is an $H_{g}^{*}$-point. This proves the claim. Note that

$$
\left\|d_{n} \chi\left(0, t_{\varepsilon_{n}}\right) \cap A_{n}\left(\varepsilon_{n}\right)\right\|_{E} \leq\left\|d_{n} \chi_{A_{n}\left(\varepsilon_{n}\right)}\right\|_{E} \rightarrow 0 .
$$

Therefore, by (4.2)

$$
\left\|d_{n} \chi\left(0, t_{\varepsilon_{n}}\right)\right\|_{E} \leq C_{E}\left\|d_{n} \chi_{\left(0, t_{\varepsilon_{n}}\right) \cap A_{n}\left(\varepsilon_{n}\right)}\right\|_{E}+C_{E}\left\|d_{n} \chi_{\left(0, t_{\varepsilon_{n}}\right) \backslash A_{n}\left(\varepsilon_{n}\right)}\right\|_{E} \rightarrow 0 .
$$

Consequently,

$$
\delta / 2 C_{E} \leq\left\|d_{n} \chi_{\left(t_{\varepsilon_{n}}, \infty\right)}\right\|_{E} \leq\left\|x^{*} \chi_{\left(t_{\varepsilon_{n}}, \infty\right)}\right\|_{E}
$$

for sufficiently large $n \in N$. Taking $y_{n}=x^{*} \chi_{\left(0, t_{\varepsilon_{n}}\right)}$, we conclude that $y_{n}=y_{n}^{*} \rightarrow x^{*}$ globally in measure and $\left\|y_{n}\right\|_{E} \rightarrow\left\|x^{*}\right\|_{E}$. On the other hand, $\left\|y_{n}-x^{*}\right\|_{E} \geq \delta / 2 C_{E}$ for sufficiently large $n \in N$. This means $x^{*}$ is not an $H_{g}^{*}$-point, a contradiction. It finishes the proof. 
Definition We say that $E$ satisfies the condition $(+)$ if for each sequence $\left(y_{n}\right)$ in $E$ with $\left(y_{n}^{*}\right)$ in $E$ the condition $\left\|y_{n}^{*}\right\|_{E} \rightarrow 0$ implies $\left\|y_{n}\right\|_{E} \rightarrow 0$.

Examples 1. Let $E=L^{p}(\varphi), p>0$ with $\varphi: R_{+} \rightarrow R_{+}$such that $\varphi \downarrow$ and $\int_{0}^{t} \varphi(s) d s<\infty$ for $t>0$. Then, applying Hardy-Littlewood inequality, we conclude that $E \in(+)$.

2. Let $E=L^{\infty}(\varphi)$ with $\varphi: R_{+} \rightarrow R_{+}$such that $\varphi \in L^{\infty}, \varphi$ is continuous on $R_{+}$ and $\varphi\left(0^{+}\right)>0$. Then $E \in(+)$. Indeed, if $\left\|y_{n}^{*}\right\|_{E} \rightarrow 0$ then $y_{n}^{*}\left(0^{+}\right) \rightarrow 0$. Thus $\left\|y_{n}\right\|_{L^{\infty}} \rightarrow 0$, whence

$$
\left\|y_{n}\right\|_{E}=\underset{t}{\operatorname{supess}} y_{n}(t) \varphi(t) \leq\left\|y_{n}\right\|_{L^{\infty}}\|\varphi\|_{L^{\infty}} \rightarrow 0 \text {. }
$$

3. We have also $L^{\infty}(t) \notin(+)$.

Proposition 4.4 Let $E$ be a quasi-Banach function space. Suppose $x=x^{*} \in E$ and $\left\|x^{*} \chi_{\left(0, a_{n}\right)}\right\|_{E} \rightarrow 0$ for any sequence $a_{n} \rightarrow 0$. If $x$ is an $H_{g}^{*}$-point of $E$ then:

(i) for each sequence $\left(d_{n}\right) \subset E_{+}$such that $d_{n}=d_{n}^{*} \leq x$ and $d_{n} \rightarrow 0$ globally in measure we have $\left\|d_{n}\right\|_{E} \rightarrow 0$.

(ii) for each sequence $\left(x_{n}\right) \subset E_{+}$such that $x \leq x_{n}=x_{n}^{*}, x_{n} \rightarrow x$ globally in measure and $\left\|x_{n}\right\|_{E} \rightarrow\|x\|_{E}$, we have $\left\|x_{n}-x\right\|_{E} \rightarrow 0$.

If, additionally, $E \in(+)$ and $E$ is $p$-convex with the constant 1 for some $p>0$, then the converse implication is true.

Proof The necessity follows from Lemma 4.3 (point (i)) and from the definition-point (ii).

The sufficiency. Suppose $0 \leq x_{n}=x_{n}^{*} \rightarrow x$ globally in measure and $\left\|x_{n}\right\|_{E} \rightarrow\|x\|_{E}$. Set

$$
A_{n}=\left\{t \in I: x(t) \geq x_{n}(t)\right\} .
$$

Then $0 \leq\left(x-x_{n}\right) \chi_{A_{n}} \leq x$ and $d_{n}:=\left[\left(x-x_{n}\right) \chi_{A_{n}}\right]^{*} \leq x$. Then $d_{n} \rightarrow 0$ globally in measure, by Lemma 3.5. By (i), we have $\left\|d_{n}\right\|_{E} \rightarrow 0$, whence $\left\|\left(x-x_{n}\right) \chi_{A_{n}}\right\|_{E} \rightarrow 0$, because $E \in(+)$. Finally, we follow as in the proof of Theorem 3.15.

Applying Lemma 3.4 we conclude immediately.

Remark 4.5 Suppose $E$ is a quasi-Banach function space which is $p$-convex with the constant 1 for some $p>0$. If $x$ is an $H_{g}$-point of $E, x_{n} \rightarrow x$ globally in measure and $\left\|x_{n}\right\|_{E} \rightarrow\|x\|_{E}$, then $\left\|x_{n} \chi_{A_{n}}\right\|_{E} \rightarrow\left\|x \chi_{A_{n}}\right\|_{E}$ for each sequence $\left(A_{n}\right)$ of measurable sets. Similarly, if $x=x^{*}$ is an $H_{g}^{*}$-point of $E, x_{n}=x_{n}^{*}, x_{n} \rightarrow x$ globally in measure and $\left\|x_{n}\right\|_{E} \rightarrow\|x\|_{E}$, then $\left\|x_{n} \chi_{A_{n}}\right\|_{E} \rightarrow\left\|x \chi_{A_{n}}\right\|_{E}$ for each sequence $\left(A_{n}\right)$ of measurable sets.

Remark 4.6 Let $E$ be a quasi-Banach function space. If $x^{*}$ is an $H_{g}^{*}$-point of $E$ then $a x^{*}$ is an $H_{g}^{*}$-point of $E$ for each $a>0$. Indeed, suppose $x_{n}=x_{n}^{*} \rightarrow a x^{*}$ globally in measure and $\left\|x_{n}\right\|_{E} \rightarrow\left\|a x^{*}\right\|_{E}$. Set $y_{n}=x_{n} / a$. Then $\left\|y_{n}\right\|_{E} \rightarrow\left\|x^{*}\right\|_{E}$ and $y_{n} \rightarrow x^{*}$ globally in measure. By the assumption, $\left\|y_{n}-x^{*}\right\|_{E} \rightarrow 0$. Thus $\left\|x_{n}-a x^{*}\right\|_{E} \rightarrow 0$. 
Lemma 4.7 Suppose $E$ is a quasi-Banach function space and $x \in E^{(*)} \backslash\{0\}$. If $x$ is an $H_{g}$-point of $E^{(*)}$ then $\left\|x^{*} \chi_{\left(0, a_{n}\right)}\right\|_{E} \rightarrow 0$ for any sequence $a_{n} \rightarrow 0$.

Proof Let $a_{n} \rightarrow 0$. We will prove that $\left\|x^{*} \chi_{\left(0, a_{n}\right)}\right\|_{E} \rightarrow 0$. Set

$$
C=\left\{t:|x(t)|>x^{*}(\infty)\right\} .
$$

We divide the proof into parts.

(a) Suppose $m(C)>0$. Then there exists a measure preserving transformation $\sigma: C \rightarrow(0, m(C))$ such that $x^{*} \circ \sigma=|x| m$-a.e. on $C$ (see Lemma 2.2 in [7]). Passing to a subsequence if necessary, we may assume that $a_{n} \leq m(C)$ for each $n$. Take a sequence $\left(A_{n}\right)$ in $C$ such that $m\left(A_{n}\right)=a_{n}$ and $x^{*} \chi_{\left(0, a_{n}\right)} \circ \sigma=|x| \chi_{A_{n}}$. Moreover,

$$
\begin{aligned}
\left\|x^{*} \chi_{\left(0, a_{n}\right)}\right\|_{E} & =\left\|\left(x^{*} \chi_{\left(0, a_{n}\right)}\right)^{*}\right\|_{E}=\left\|\left(x^{*} \chi_{\left(0, a_{n}\right)} \circ \sigma\right)^{*}\right\|_{E} \\
& =\left\|\left(|x| \chi_{A_{n}}\right)^{*}\right\|_{E}=\left\||x| \chi_{A_{n}}\right\|_{E^{(*)}} .
\end{aligned}
$$

By the assumption, $|x|$ is an $H_{g}$-point of $E^{(*)}$ (see Theorem 3.9, this part works without the assumption that $E$ is $L$-convex ). Then $\left\||x| \chi_{A_{n}}\right\|_{E^{(*)}} \rightarrow 0$, by Lemma 3.7.

(b) Assume that $m(C)=0$. Then $I=(0, \infty)$ and $x^{*}=c \chi_{I}$.

(b1) If $x \chi_{A}=c$ for some set $A$ with $m(\mathrm{~A})>0$, we choose sequence $\left(A_{n}\right)$ in $A$ such that $m\left(A_{n}\right)=a_{n}$ and

$$
\left\|x^{*} \chi_{\left(0, a_{n}\right)}\right\|_{E}=\left\|\left(|x| \chi_{A_{n}}\right)^{*}\right\|_{E}=\left\||x| \chi_{A_{n}}\right\|_{E^{(*)}} \rightarrow 0 .
$$

(b2) Assume that $x(t)<c$ for all $t \in I$. Then we find a sequence $\left(A_{n}\right)$ such that $m\left(A_{n}\right)=a_{n}$ and $|x| \chi_{A_{n}} \rightarrow c \chi_{A_{n}}$ uniformly, whence $\left(|x| \chi_{A_{n}}\right)^{*} \rightarrow x^{*} \chi_{\left(0, a_{n}\right)}$ uniformly. Moreover,

$$
\left\|\left(|x| \chi_{A_{n}}\right)^{*}\right\|_{E}=\left\||x| \chi_{A_{n}}\right\|_{E^{(*)}} \rightarrow 0
$$

as above. Since $\chi_{I} \in E$, we get

$$
\left\|x^{*} \chi_{\left(0, a_{n}\right)}\right\|_{E} \leq C_{E}\left(\left\|x^{*} \chi_{\left(0, a_{n}\right)}-\left(|x| \chi_{A_{n}}\right)^{*}\right\|_{E}+\left\|\left(|x| \chi_{A_{n}}\right)^{*}\right\|_{E}\right) \rightarrow 0 .
$$

Definition Let $E$ be a quasi-Banach function space. We say that an element $x=x^{*} \in$ $E$ satisfies the condition $(*)$, we write $x \in(*)$, if for all $\left(y_{n}\right), y$ in $E$ the conditions $0 \leq y \leq x, 0 \leq y_{n} \leq x, y_{n} \rightarrow y$ globally in measure imply that $\left\|y_{n}\right\|_{E} \rightarrow\|y\|_{E}$.

Example 4.8 Suppose $E$ is a quasi-Banach function space which is $p$-convex with the constant 1 for some $p>0$ and $x=x^{*} \in E$. Note that we may apply Lemma 3.4. Consequently, 
(i) If $x \in E_{a}$, then $x \in(*)$. Indeed, taking elements $y, y_{n}$ as in the definition we get $0 \leq\left|y_{n}-y\right| \leq x,\left|y_{n}-y\right| \rightarrow 0$ globally in measure. Since $x \in E_{a}$, applying Theorem 2.1 we obtain $\left\|y_{n}-y\right\| \rightarrow 0$. Thus, $\left\|y_{n}\right\|_{E} \rightarrow\|y\|_{E}$, by Lemma 3.4.

(ii) Suppose $E \in(+)$. If $x$ is an $H_{g}^{*}$-point of $E,\left\|x^{*} \chi_{\left(0, a_{n}\right)}\right\|_{E} \rightarrow 0$ for any sequence $a_{n} \rightarrow 0$, then $x \in(*)$ (see Remark 4.10 below).

(iii) If $x$ is an $H_{g}$-point and $E$ is symmetric then, by Lemma 3.14, $x \in(*)$.

Remark 4.2 shows that in the following theorem:

(1) the implication (i) $\Rightarrow$ (ii) is not true in general without the assumption that $\left\|x^{*} \chi\left(0, a_{n}\right)\right\|_{E} \rightarrow 0$ for any sequence $a_{n} \rightarrow 0$

(2) two assumptions in condition (i) below are independent.

Theorem 4.9 Let $E$ be a quasi-Banach function space and $x \in E^{(*)} \backslash\{0\}$. Consider the following statements:

(i) (a) $\left\|x^{*} \chi_{\left(0, a_{n}\right)}\right\|_{E} \rightarrow 0$ for any sequence $a_{n} \rightarrow 0$.

(b) The point $x^{*}$ is an $H_{g}^{*}$-point of $E$.

(ii) The point $x$ is an $H_{g}$-point of $E^{(*)}$.

(1) Suppose $E$ is $p$-convex with the constant 1 for some $p>0$. Assume that $x^{*} \in(*)$ when $\chi_{I} \notin E$. Then (i) $\Rightarrow$ (ii).

(2) The implication (ii) $\Rightarrow$ (i) (a) is true. If, additionally, $E \in(+)$ and $x^{*}(\infty)=$ 0 , then (ii) $\Rightarrow$ (i) (b).

Remark 4.10 If we assume additionally in the implication (i) $\Rightarrow$ (ii) that $E \in(+)$ then the condition $x^{*} \in(*)$ follows from (i) automatically. Indeed, suppose condition (i) holds, $E$ is $p$-convex with the constant 1 for some $p>0$ and $E \in(+)$. We prove that $x^{*} \in(*)$. Let $0 \leq y \leq x^{*}, 0 \leq y_{n} \leq x^{*}, y_{n} \rightarrow y$ globally in measure. Then $\left|y_{n}-y\right| \leq x^{*}$ and $\left|y_{n}-y\right| \rightarrow 0$ globally in measure. Thus $\left(y_{n}-y\right)^{*} \rightarrow 0$ globally in measure by Lemma 3.5. By Lemma 4.3 and the assumption that $x^{*}$ is an $H_{g}^{*}$-point of $E,\left\|\left(y_{n}-y\right)^{*}\right\|_{E} \rightarrow 0$ and, since $E \in(+),\left\|y_{n}-y\right\|_{E} \rightarrow 0$. Finally, Lemma 3.4 implies that $\left\|y_{n}\right\|_{E} \rightarrow\|y\|_{E}$.

Proof of Theorem (i) $\Rightarrow$ (ii). Suppose $\left(x_{n}\right) \subset E^{(*)}, x_{n} \rightarrow x$ globally in measure and $\left\|x_{n}\right\|_{E^{(*)}} \rightarrow\|x\|_{E^{(*)}}$. Thus $\left\|x_{n}^{*}\right\|_{E} \rightarrow\left\|x^{*}\right\|_{E}$. We need to show that

$$
\left\|x_{n}-x\right\|_{E^{(*)}} \rightarrow 0 .
$$

Set $\varepsilon>0$. We divide the proof into several parts.

I. Suppose $\chi_{I} \in E$. Denote

$$
A_{n}(\varepsilon)=\left\{t \in I:\left|x_{n}(t)-x(t)\right|>\frac{\varepsilon}{4 C_{E^{(*)}}\left\|\chi_{I}\right\|_{E}}\right\} .
$$

Then

$$
\left\|x_{n}-x\right\|_{E^{(*)}} \leq C_{E^{(*)}}\left[\left\|\left(x_{n}-x\right) \chi_{A_{n}(\varepsilon)}\right\|_{E^{(*)}}+\left\|\left(x_{n}-x\right) \chi_{A_{n}^{\prime}(\varepsilon)}\right\|_{E^{(*)}}\right],
$$


where $A_{n}^{\prime}(\varepsilon)=I \backslash A_{n}(\varepsilon)$. Note that $\left\|\left(x_{n}-x\right) \chi_{A_{n}^{\prime}(\varepsilon)}\right\|_{E^{(*)}}<\varepsilon / 4 C_{E^{(*)}}$ for each $n$. Moreover,

$$
\left\|\left(x_{n}-x\right) \chi_{A_{n}(\varepsilon)}\right\|_{E^{(*)}} \leq C_{E^{(*)}}\left[\left\|\left(x_{n} \chi_{A_{n}(\varepsilon)}\right)^{*}\right\|_{E}+\left\|\left(x \chi_{A_{n}(\varepsilon)}\right)^{*}\right\|_{E}\right] .
$$

By the assumption $m\left(A_{n}(\varepsilon)\right) \rightarrow 0$. Then $\left\|x^{*} \chi\left(0, m\left(A_{n}(\varepsilon)\right)\right)\right\|_{E} \rightarrow 0$, by (i) (a). Note that $\left(x \chi_{A_{n}(\varepsilon)}\right)^{*} \leq x^{*} \chi_{\left(0, m\left(A_{n}(\varepsilon)\right)\right)}$. Consequently, there is $N_{0}$ such that $\left\|\left(x \chi_{A_{n}(\varepsilon)}\right)^{*}\right\|_{E}<\varepsilon /\left(4 C_{E^{(*)}}^{2}\right)$ for $n \geq N_{0}$. Similarly, $\left(x_{n} \chi_{A_{n}(\varepsilon)}\right)^{*} \leq x_{n}^{*} \chi_{\left(0, m\left(A_{n}(\varepsilon)\right)\right)}$. Note that $x_{n}^{*} \rightarrow x^{*}$ globally in measure (see Lemma 3.5). By Remark 4.5, $\left\|x_{n}^{*} \chi_{\left(0, m\left(A_{n}(\varepsilon)\right)\right)}\right\|_{E} \rightarrow\left\|x^{*} \chi_{\left(0, m\left(A_{n}(\varepsilon)\right)\right)}\right\|_{E}$, since $x^{*}$ is an $H_{g}^{*}$-point. Thus there is $N_{1}$ such that $\left\|\left(x_{n} \chi_{A_{n}(\varepsilon)}\right)^{*}\right\|_{E}<\varepsilon /\left(4 C_{E^{(*)}}^{2}\right)$ for $n \geq N_{1}$. Finally, $\left\|x_{n}-x\right\|_{E^{(*)}}<\varepsilon$ for $n \geq \max \left\{N_{0}, N_{1}\right\}$.

II. Assume that $\chi_{I} \notin E$. It means $u^{*}(\infty)=0$ for each $u \in E^{(*)}$. Denote

$$
A_{k}=\{t \in I:|x(t)|<1 / k\} \text { and } \widetilde{A_{k}}=\left\{t \in I: x^{*}(t)<2 / k\right\} .
$$

Note that $\left(x \chi_{A_{k}}\right)^{*} \leq x^{*}$ and $\left(x \chi_{A_{k}}\right)^{*} \rightarrow 0$ globally in measure, whence by Lemma 4.3, $\left\|\left(x \chi_{A_{k}}\right)^{*}\right\|_{E} \rightarrow 0$. Moreover, $\left\|x^{*} \chi_{\widetilde{A_{k}}}\right\|_{E} \rightarrow 0$ by Lemma 4.1. Thus there is $k_{0}$ such that

$$
\left\|\left(x \chi_{A_{k_{0}}}\right)^{*}\right\|_{E} \leq \varepsilon /\left(16 C_{E^{(*)}}^{3}\right) \text { and }\left\|x^{*} \chi_{\widetilde{A_{k_{0}}}}\right\|_{E} \leq \frac{\varepsilon}{16 C_{E^{(*)}}^{3} C_{E}} .
$$

1. We prove that there is $N_{0} \in N$ such that

$$
\left\|\left(x_{n}-x\right) \chi_{A_{k_{0}}}\right\|_{E^{(*)}} \leq \varepsilon /\left(2 C_{E^{(*)}}\right)
$$

for all $n \geq N_{0}$. Set

$$
\begin{aligned}
& A_{k_{0}}^{n}=\left\{t \in A_{k_{0}}:\left|x_{n}(t)-x(t)\right| \geq 1 / k_{0}\right\} \text { and } \\
& B_{k_{0}}^{n}=\left\{t \in A_{k_{0}}:\left|x_{n}(t)-x(t)\right|<1 / k_{0}\right\} .
\end{aligned}
$$

Then $m\left(A_{k_{0}}^{n}\right) \rightarrow 0$. By the assumption (a), there is $N_{1}$ such that

$$
\left\|\left(x \chi_{A_{k_{0}}^{n}}\right)^{*}\right\|_{E} \leq\left\|x^{*} \chi\left(0, m\left(A_{k_{0}}^{n}\right)\right]\right\|_{E} \leq \varepsilon /\left(16 C_{E^{(*)}}^{3}\right)
$$

for $n \geq N_{1}$. Note that $x_{n}^{*} \rightarrow x^{*}$ globally in measure (see Lemma 3.5). Since $\left(x_{n} \chi_{A_{k_{0}}^{n}}\right)^{*} \leq x_{n}^{*} \chi_{\left(0, m\left(A_{k_{0}}^{n}\right)\right.}$ and $x^{*}$ is an $H_{g}^{*}$-point, by Remark 4.5, there is $N_{2} \geq N_{1}$ such that

$$
\left\|\left(x_{n} \chi_{A_{k_{0}}^{n}}\right)^{*}\right\|_{E}<\varepsilon /\left(8 C_{E^{(*)}}^{3}\right)
$$


for $n \geq N_{2}$. Moreover, by (4.3),

$$
\left\|\left(x \chi_{B_{k_{0}}^{n}}\right)^{*}\right\|_{E} \leq\left\|\left(x \chi_{A_{k_{0}}}\right)^{*}\right\|_{E} \leq \varepsilon /\left(16 C_{E^{(*)}}^{3} C_{E}\right) .
$$

Now we estimate the norm $\left\|\left(x_{n} \chi_{B_{k_{0}}^{n}}\right)^{*}\right\|_{E}$. Note that $\left|x_{n}(t)\right|<2 / k_{0}$ for $t \in B_{k_{0}}^{n}$, whence

$$
\left(x_{n} \chi_{B_{k_{0}}^{n}}\right)^{*} \chi_{I \backslash \widetilde{A_{k_{0}}}} \leq x^{*} \chi_{I \backslash \widetilde{A_{k_{0}}}} \text { and }\left(x \chi_{B_{k_{0}}^{n}}\right)^{*} \chi_{I \backslash \widetilde{A_{k_{0}}}} \leq x^{*} \chi_{I \backslash \widetilde{A_{k_{0}}}}
$$

Moreover, $x_{n} \chi_{B_{k_{0}}^{n}} \rightarrow x \chi_{B_{k_{0}}^{n}}$ globally in measure, so $\left(x_{n} \chi_{B_{k_{0}}^{n}}\right)^{*} \rightarrow\left(x \chi_{B_{k_{0}}^{n}}\right)^{*}$ globally in measure (see Lemma 3.5). The assumption $x^{*} \in(*)$ gives $\left\|\left(x_{n} \chi_{B_{k_{0}}^{n}}\right)^{*} \chi_{I \backslash \widetilde{A_{k_{0}}}}\right\|_{E} \rightarrow$ $\left\|\left(x \chi_{B_{k_{0}}^{n}}\right)^{*} \chi_{I \backslash \widetilde{A_{k_{0}}}}\right\|_{E}$. Thus, by (4.6), there is $N_{3}$ such that

$$
\left\|\left(x_{n} \chi_{B_{k_{0}}^{n}}\right)^{*} \chi_{I \backslash \widetilde{A_{k_{0}}}}\right\|_{E} \leq \frac{\varepsilon}{8 C_{E^{(*)}}^{3} C_{E}}
$$

for $n \geq N_{3}$. Moreover, $\left(x_{n} \chi_{B_{k_{0}}^{n}}\right)^{*} \chi_{\widetilde{A_{k_{0}}}} \leq x_{n}^{*} \chi_{\widetilde{A_{k_{0}}}}$ and $\left\|x_{n}^{*} \chi_{\widetilde{A_{k_{0}}}}\right\|_{E} \rightarrow\left\|x^{*} \chi_{\widetilde{A_{k_{0}}}}\right\|_{E}$, by Remark 4.5. Consequently, by (4.3), there is $N_{4}$ such that

$$
\left\|\left(x_{n} \chi_{B_{k_{0}}^{n}}\right)^{*} \chi \widetilde{A_{k_{0}}}\right\|_{E} \leq\left\|x_{n}^{*} \chi_{\widetilde{A_{k_{0}}}}\right\|_{E} \leq \frac{\varepsilon}{8 C_{E^{(*)}}^{3} C_{E}}
$$

for $n \geq N_{4}$. Summing up the above conditions (4.3), (4.5), ( 4.7) and (4.8), taking $N_{0}=\max \left\{N_{1}, N_{2}, N_{3}, N_{4}\right\}$ we obtain

$$
\begin{aligned}
& \left\|\left(x_{n}-x\right) \chi_{A_{k_{0}}}\right\|_{E^{(*)}} \leq C_{E^{(*)}}\left[\left\|x_{n} \chi_{A_{k_{0}}}\right\|_{E^{(*)}}+\left\|x \chi_{A_{k_{0}}}\right\|_{E^{(*)}}\right] \\
& \leq \varepsilon /\left(8 C_{E^{(*)}}\right)+C_{E^{(*)}}^{2}\left[\left\|x_{n} \chi_{A_{k_{0}}^{n}}\right\|_{E^{(*)}}+\left\|x_{n} \chi_{B_{k_{0}}^{n}}\right\|_{E^{(*)}}\right] \\
& \leq \varepsilon /\left(4 C_{E^{(*)}}\right)+C_{E^{(*)}}^{2}\left\|x_{n} \chi_{B_{k_{0}}^{n}}\right\|_{E^{(*)}} \\
& =\varepsilon /\left(4 C_{E^{(*)}}\right)+C_{E^{(*)}}^{2}\left\|\left(x_{n} \chi_{B_{k_{0}}^{n}}\right)^{*}\right\|_{E} \\
& \leq \varepsilon /\left(4 C_{E^{(*)}}\right)+C_{E^{(*)}}^{2} C_{E}\left[\left\|\left(x_{n} \chi_{B_{k_{0}}^{n}}\right)^{*} \chi_{\widetilde{A_{k_{0}}}}\right\|_{E}+\left\|\left(x_{n} \chi_{B_{k_{0}}^{n}}\right)^{*} \chi_{I \backslash \widetilde{A_{k_{0}}}}\right\|_{E}\right] \\
& \leq \varepsilon /\left(4 C_{E^{(*)}}\right)+\varepsilon /\left(4 C_{E^{(*)}}\right)=\varepsilon /\left(2 C_{E^{(*)}}\right)
\end{aligned}
$$

for $n \geq N_{0}$.

2. Now we estimate $\left\|\left[\left(x_{n}-x\right) \chi_{A_{k_{0}}^{\prime}}\right]^{*}\right\|_{E}$, where $A_{k_{0}}^{\prime}=I \backslash A_{k_{0}}$. Set

$$
C_{n}=\left\{t \in A_{k_{0}}^{\prime}:\left|x_{n}(t)\right| \leq 2|x(t)|\right\} \text { and } D_{n}=\left\{t \in A_{k_{0}}^{\prime}:\left|x_{n}(t)\right|>2|x(t)|\right\} \text {. }
$$


Note again that

$$
\left\|\left(x_{n}-x\right) \chi_{A_{k_{0}}^{\prime}}\right\|_{E^{(*)}} \leq C_{E^{(*)}}\left\{\left\|\left[\left(x_{n}-x\right) \chi_{C_{n}}\right]^{*}\right\|_{E}+\left\|\left[\left(x_{n}-x\right) \chi_{D_{n}}\right]^{*}\right\|_{E}\right\} .
$$

We have $\left|\left(x_{n}(t)-x(t)\right) \chi_{C_{n}}\right| \leq 3|x(t)| \chi_{C_{n}} \leq 3|x(t)|$, whence $\left(\left(x_{n}-x\right) \chi_{C_{n}}\right)^{*} \leq$ $3 x^{*}$. Since $\left(x_{n}-x\right) \chi_{C_{n}} \rightarrow 0$ globally in measure, so $\left(\left(x_{n}-x\right) \chi_{C_{n}}\right)^{*} \rightarrow 0$ globally in measure (see Lemma 3.5). By Lemma 4.3, $\left\|\left(\left(x_{n}-x\right) \chi_{C_{n}}\right)^{*}\right\|_{E} \rightarrow 0$, because $3 x^{*}$ is an $H_{g}^{*}$-point (see Remark 4.6). Thus, there is $N_{5}$ such that

$$
\left\|\left(\left(x_{n}-x\right) \chi_{C_{n}}\right)^{*}\right\|_{E}<\varepsilon /\left(8 C_{E^{(*)}}^{2}\right) \text { for } n \geq N_{5}
$$

We have $\left|\left(x_{n}-x\right)(t)\right| \geq|x(t)| \geq 1 / k_{0}$ for $t \in D_{n}$. Thus $m\left(D_{n}\right) \rightarrow 0$. Since $\left(x \chi_{D_{n}}\right)^{*} \leq x^{*}$ and $\left(x \chi_{D_{n}}\right)^{*} \rightarrow 0$ globally in measure, there is $N_{6}$ such that $\left\|\left(x \chi_{D_{n}}\right)^{*}\right\|_{E}<\varepsilon /\left(8 C_{E^{(*)}}^{3}\right)$ for $n \geq N_{6}$, by Lemma 4.3. Furthermore, $\left[x_{n} \chi_{D_{n}}\right]^{*} \leq$ $x_{n}^{*} \chi_{\left(0, m\left(D_{n}\right)\right)}$ and $\left\|x^{*} \chi_{\left(0, m\left(D_{n}\right)\right)}\right\| \rightarrow 0$ by the assumption (i) (a). Consequently, $\left\|x_{n}^{*} \chi_{\left(0, m\left(D_{n}\right)\right)}\right\| \rightarrow 0$, by Remark 4.5. Therefore, there is $N_{7}$ such that $\left\|\left(x_{n} \chi_{D_{n}}\right)^{*}\right\|_{E}$ $<\varepsilon /\left(8 C_{E^{(*)}}^{3}\right)$ for $n \geq N_{7}$. Consequently, for $n \geq \max \left\{N_{5}, N_{6}, N_{7}\right\}$, we have

$$
\begin{aligned}
\left\|\left(x_{n}-x\right) \chi_{A_{k_{0}}^{\prime}}\right\|_{E^{(*)}} & \leq \varepsilon /\left(8 C_{E^{(*)}}\right)+C_{E^{(*)}}\left\|\left[\left(x_{n}-x\right) \chi_{D_{n}}\right]^{*}\right\|_{E} \\
& \leq \varepsilon /\left(8 C_{E^{(*)}}\right)+C_{E^{(*)}}^{2}\left[\left\|\left[x \chi_{D_{n}}\right]^{*}\right\|_{E}+\left\|\left[x_{n} \chi_{D_{n}}\right]^{*}\right\|_{E}\right] \\
& \leq \varepsilon /\left(2 C_{E^{(*)}}\right) .
\end{aligned}
$$

Summing up cases 1 and 2 we obtain

$$
\begin{aligned}
\left\|x_{n}-x\right\|_{E^{(*)}} & \leq C_{E^{(*)}}\left(\left\|\left(x_{n}-x\right) \chi_{A_{k_{0}}}\right\|_{E^{(*)}}+\left\|\left(x_{n}-x\right) \chi_{A_{k_{0}}^{\prime}}\right\|_{E^{(*)}}\right) \\
& <\varepsilon \text { for } n \geq \max \left\{N_{1}, \ldots, N_{7}\right\} .
\end{aligned}
$$

(ii) $\Rightarrow$ (i). The condition $\left\|x^{*} \chi_{\left(0, a_{n}\right)}\right\|_{E} \rightarrow 0$ for any sequence $a_{n} \rightarrow 0$ follows from Lemma 4.7.

We prove the condition (b). Let $\left(x_{n}\right) \subset E, x_{n}=x_{n}^{*}$ and $x_{n}^{*} \rightarrow x^{*}$ globally in measure and $\left\|x_{n}^{*}\right\|_{E} \rightarrow\left\|x^{*}\right\|_{E}$. Since $x^{*}(\infty)=0$, there is a measure preserving transformation:

(I) $\gamma: I \rightarrow I$ such that $x^{*} \circ \gamma=|x|$ a.e. when $m(\operatorname{supp} x)<\infty$,

(II) $\gamma: \operatorname{supp} x \rightarrow(0, \infty)$ such that $x^{*} \circ \gamma=|x|$ a.e. on supp $x$ when $m(\operatorname{supp} x)=\infty$ (see Lemma 2 in [19]).

Set $\tilde{x_{n}}=x_{n} \circ \gamma$ in the case (I) and

$$
\widetilde{x_{n}}= \begin{cases}x_{n}(\gamma(t)) & \text { if } t \in \operatorname{supp} x \\ 0 & \text { if } t \notin \operatorname{supp} x\end{cases}
$$


in the case (II). Then $\tilde{x_{n}} \rightarrow|x|$ in measure. Moreover,

$$
\left\|x_{n}\right\|_{E}=\left\|x_{n}^{*}\right\|_{E}=\left\|\left(x_{n} \circ \gamma\right)^{*}\right\|_{E}=\left\|\left(\tilde{x_{n}}\right)^{*}\right\|_{E}=\left\|\tilde{x_{n}}\right\|_{E^{(*)}},
$$

whence $\left\|\tilde{x}_{n}\right\|_{E^{(*)}} \rightarrow\||x|\|_{E^{(*)}}$. Since $x$ is an $H_{g}$-point of $E^{(*)}$, by Theorem 3.9, $|x|$ is an $H_{g}$-point of $E^{(*)}$ (note that this implication is true without the assumption that $E$ is $L$-convex). In consequence,

$$
\left\|\left(x_{n}^{*}-x^{*}\right)^{*}\right\|_{E}=\left\|\left(\left(x_{n}^{*}-x^{*}\right) \circ \gamma\right)^{*}\right\|_{E}=\left\|\left(\tilde{x_{n}}-|x|\right)^{*}\right\|_{E}=\left\|\tilde{x_{n}}-|x|\right\|_{E^{(*)}} \rightarrow 0 .
$$

Since $E \in(+)$, we have $\left\|x_{n}^{*}-x^{*}\right\|_{E} \rightarrow 0$ as desired.

Remark 4.11 In Theorem 4.9 the implication (ii) $\Rightarrow$ (i) (b) is not true in general without the assumption that $E \in(+)$. Let $E=L^{1}(0,1 / 2] \oplus L^{\infty}(1 / 2,1)$ on $I=(0,1)$ with the norm

$$
\|x\|_{E}=\left\|x \chi_{(0,1 / 2]}\right\|_{L^{1}}+\left\|x \chi_{(1 / 2,1)}\right\|_{L^{\infty}} .
$$

Clearly, $E \notin(+)$, it is enough to consider $x_{n}=\chi_{[1 / 2,1 / 2+1 / n]}$. We need to show that $\|\cdot\|_{E^{(*)}}$ is a quasi-norm or equivalently that there is a constant $C>0$ such that

$$
\left\|D_{2} x^{*}\right\|_{E} \leq C\left\|x^{*}\right\|_{E} \text { for all } x^{*} \in E
$$

Let $x=x^{*} \in E$. Then

$$
\int_{0}^{1 / 2}\left(D_{2} x\right)(s) \chi_{(0,1 / 2)}(s) d s=\int_{0}^{1 / 2} x(s / 2) d s=2 \int_{0}^{1 / 4} x(s) d s \leq 2\|x\|_{E} .
$$

Moreover, $D_{2} x=\left(D_{2} x\right)^{*}$,

$$
\left\|\left(D_{2} x\right) \chi_{(1 / 2,1]}\right\|_{L^{\infty}} \leq D_{2} x(1 / 2)=x(1 / 4)
$$

and

$$
\|x\|_{E} \geq\left\|x \chi_{(0,1 / 2]}\right\|_{L^{1}} \geq\left\|x \chi_{(0,1 / 4]}\right\|_{L^{1}} \geq x(1 / 4) / 4 .
$$

Thus $\left\|\left(D_{2} x\right) \chi_{(1 / 2,1]}\right\|_{L^{\infty}} \leq 4\|x\|_{E}$. Consequently,

$$
\left\|D_{2} x\right\|_{E}=\left\|\left(D_{2} x\right) \chi_{(0,1 / 2]}\right\|_{L^{1}}+\left\|\left(D_{2} x\right) \chi_{(1 / 2,1]}\right\|_{L^{\infty}} \leq 6\|x\|_{E} .
$$

Set

$$
x=\chi_{(0,1 / 2]}+\chi_{(3 / 4,1)} .
$$

Then $x^{*}=\chi_{(0,3 / 4]}$ and $\|x\|_{E^{(*)}}=\left\|x^{*}\right\|_{E}=3 / 2$. Taking $x_{n}=\chi_{(0,3 / 4+1 / n]}$, we have $x_{n}=x_{n}^{*},\left\|x_{n}\right\|_{E}=3 / 2$ and $x_{n}^{*} \rightarrow x^{*}$ globally in measure. However, $\left\|x_{n}^{*}-x^{*}\right\|_{E}=1$, whence $x^{*}$ is not an $H_{g}^{*}$-point of $E$. 
We will prove that $x$ is an $H_{g}$-point of $E^{(*)}$. Let $\left(x_{n}\right) \subset E^{(*)}$ be a sequence such that $x_{n} \rightarrow x$ globally in measure and $\left\|x_{n}\right\|_{E^{(*)}} \rightarrow\|x\|_{E^{(*)}}$. Let $\varepsilon>0$. Denote

$$
A_{n}(\varepsilon)=\left\{t \in I:\left|x_{n}(t)-x(t)\right|>\frac{\varepsilon}{4\left\|\chi_{I}\right\|_{E^{(*)}}}\right\} \text {. }
$$

Note that

$$
\left\|\left(x_{n}-x\right) \chi_{I \backslash A_{n}(\varepsilon)}\right\|_{E^{(*)}} \leq \varepsilon / 4 \text {. }
$$

Moreover,

$$
\left\|\left(x_{n}-x\right) \chi_{A_{n}(\varepsilon)}\right\|_{E^{(*)}} \leq 6\left(\left\|x_{n} \chi_{A_{n}(\varepsilon)}\right\|_{E^{(*)}}+\left\|x \chi_{A_{n}(\varepsilon)}\right\|_{E^{(*)}}\right) .
$$

Since $m\left(A_{n}(\varepsilon)\right) \rightarrow 0$, so relabelling, if necessary, we assume that $m\left(A_{n}(\varepsilon)\right) \leq$ $1 / 2$ for each $n$ and consequently both norms above reduce to $L^{1}(0,1 / 2]$. Then $\left\|x \chi_{A_{n}(\varepsilon)}\right\|_{E^{(*)}} \rightarrow 0$. Set

$$
A_{n}^{1}=\left\{t \in A_{n}(\varepsilon): x_{n}(t) \leq 2\right\} \text { and } A_{n}^{2}=\left\{t \in A_{n}(\varepsilon): x_{n}(t)>2\right\} \text {. }
$$

Then $\left\|x_{n} \chi_{A_{n}^{1}}\right\|_{E^{(*)}} \rightarrow 0$. Furthermore, $x_{n}^{*} \rightarrow x^{*}$ globally in measure, by Lemma 3.5. Consequently, $x_{n}^{*}\left(t_{0}\right) \rightarrow 1$ for each $0<t_{0}<3 / 4$. Thus $\left\|x_{n} \chi_{A_{n}^{2}}\right\|_{E^{(*)}} \rightarrow 0$ because otherwise we would get a contradiction with $\left\|x_{n}^{*}\right\|_{E} \rightarrow\left\|x^{*}\right\|_{E}$. Summing up, $\left\|x_{n}-x\right\|_{E^{(*)}} \leq \varepsilon$ for sufficiently large $n$.

If $E$ is a symmetric Banach function space then $E^{(*)} \equiv E$ and all assumptions of Theorem 4.9 are satisfied. Therefore, from Theorem 4.9 and Remark 4.10, we get immediately.

Corollary 4.12 Suppose $E$ is a symmetric Banach function space.

(i) If $x^{*}$ is an $H_{g}^{*}$-point of $E$ and $\left\|x^{*} \chi_{\left(0, a_{n}\right)}\right\|_{E} \rightarrow 0$ for any sequence $a_{n} \rightarrow 0$ then $x$ is an $H_{g}$-point of $E$.

(ii) The converse implication is true if we assume additionally that $x^{*}(\infty)=0$.

Remark 4.13 The point (i) in above Corollary is an essential generalization of Theorem 3.5 in [8], because we don't need the assumption that $x \in E_{a}$. Moreover, our assumption of (i) is weaker than $x^{*}$ is an $H_{g}$-point of $E$ which is used in Theorem 3.5 in [8].

It is known that the following useful equivalence is true in order continuous symmetric Banach function space: $\left\|x_{n}-x\right\| \rightarrow 0$ if and only if $x_{n} \rightarrow x$ globally in measure and $\left\|x_{n}^{*}-x^{*}\right\| \rightarrow 0$ (see Corollary 1.6 in [6]). Later the same result has been proved under a weaker assumption that $x \in E_{a}$ ([10, Proposition 2.4]). Now we will show that such a characterization is also true under the assumptions that $x^{*}$ is an $H_{g}^{*}$-point of $E$ and $\left\|x^{*} \chi_{\left(0, a_{n}\right)}\right\|_{E} \rightarrow 0$ for any sequence $a_{n} \rightarrow 0$. Recall that properties $O C$ and $H_{g}$ are independent (see the discussion in [8], Section 5). 
Proposition 4.14 Suppose $E$ is a symmetric Banach function space, $x^{*}$ is an $H_{g}^{*}$-point of $E$ and $\left\|x^{*} \chi_{\left(0, a_{n}\right)}\right\|_{E} \rightarrow 0$ for any sequence $a_{n} \rightarrow 0$. The following statements are equivalent:

(i) $\left\|x_{n}-x\right\|_{E} \rightarrow 0$.

(ii) $x_{n} \rightarrow x$ globally in measure and $\left\|x_{n}^{*}-x^{*}\right\|_{E} \rightarrow 0$.

Proof The implication (i) $\Rightarrow$ (ii). Suppose $\left\|x_{n}-x\right\|_{E} \rightarrow 0$. Then, of course, $x_{n} \rightarrow x$ globally in measure, since $E$ is symmetric. Moreover, $\left\|x_{n}^{*}\right\|_{E}=\left\|x_{n}\right\|_{E} \rightarrow\|x\|_{E}=$ $\left\|x^{*}\right\|_{E}$. On the other hand, $x_{n}^{*} \rightarrow x^{*}$ globally in measure, by Lemma 3.5. Thus $\left\|x_{n}^{*}-x^{*}\right\|_{E} \rightarrow 0$ because $x^{*}$ is an $H_{g}^{*}$-point of $E$.

The implication (ii) $\Rightarrow$ (i). By the assumption, $x_{n} \rightarrow x$ globally in measure and $\left\|x_{n}^{*}\right\|_{E} \rightarrow\left\|x^{*}\right\|_{E}$. Applying the proof of Theorem 4.9, the implication (i) $\Rightarrow$ (ii), we conclude that $\left\|x_{n}-x\right\|_{E} \rightarrow 0$.

Remark 4.15 Notice that if $x^{*}$ is an $H_{g}$-point of $E$ then the assumptions of the above proposition are satisfied. Moreover, if $x^{*}(\infty)=0$, we may replace the assumptions in the above proposition by $x$ is an $H_{g}$-point of $E$ (see Corollary 4.12).

Recall that $0 \leq x \in E$ is called a point of upper monotonicity ( $x$ is a $U M$-point briefly) of $E$ whenever for each $y \geq x$ and $y \neq x$ we have $\|y\|>\|x\|$ (see for example [25]). The space $E$ is called strictly monotone $(E \in(S M))$ provided each point $x \in E_{+}$is a $U M$-point of $E$.

Corollary 4.16 (i) Suppose $E$ and $E^{(*)}$ are Banach function spaces. Let $E \in(+)$ and $0 \leq x \in E^{(*)}$. The following statements are equivalent:

(i) The point $x$ is an $H_{l}$-point of $E^{(*)}$.

(ii) The point $x$ is a point of order continuity of $E^{(*)}, x$ is an $H_{g}$-point of $E^{(*)}$ and $x$ is a $U M$-point of $E^{(*)}$.

(iii) The point $x^{*}$ is an $O C^{*}$ point of $E, x^{*}(\infty)=0, x^{*}$ is an $H_{g}^{*}$-point of $E$ and $x^{*}$ is an $U M^{*}$-point of $E$.

Proof The equivalence (i) $\Leftrightarrow$ (ii) comes from Theorem 3.10 from [8]. The equivalence (ii) $\Leftrightarrow$ (iii) follows from Theorem 3.8, Theorem 3.9 in [25] and Theorem 4.9. Note that if $x^{*}$ is an $O C^{*}$ in $E$ then $\left\|x^{*} \chi_{\left(0, a_{n}\right)}\right\|_{E} \rightarrow 0$ for any sequence $a_{n} \rightarrow 0$.

From Theorem 4.9, Remark 4.10 and the above Corollary we conclude

Corollary 4.17 (i) Suppose $E$ is a quasi-Banach function space which is p-convex with the constant 1 for some $p>0$. Let $E \in(+)$ and $\chi_{(0, \infty)} \notin E$. Then the following statements are equivalent:

(a) The symmetrization $E^{(*)}$ has the property $H_{g}$ and $x^{*} \in(*)$ for all $x=x^{*} \in E$.

(b) The space $E$ has the property $H_{g}^{*}$ and $\left\|x^{*} \chi_{\left(0, a_{n}\right)}\right\|_{E} \rightarrow 0$ for each $x^{*} \in E$ and any sequence $a_{n} \rightarrow 0$.

(ii) Assume that $E$ and $E^{(*)}$ are Banach function spaces. Let $E \in(+)$. The symmetrization $E^{(*)}$ has the property $H_{l}$ if and only if $E \in\left(O C^{*}\right), \chi_{(0, \infty)} \notin E$, $E \in\left(H_{g}^{*}\right)$ and $E \in\left(S M^{*}\right)$. 


\section{Kadec-Klee properties in Marcinkiewicz spaces $M_{\phi}^{(*)}$}

Proposition 5.1 The Marcinkiewicz space $M_{\phi}^{(*)}$ has no $H_{g}$ points for any quasiconcave function $\phi$.

Proof Let $0 \neq x \in M_{\phi}^{(*)}$. Denote

$$
a_{n}=\sup _{t \in(0,1 / n]} x^{*}(t) \phi(t) \text { for } n=2,3 \ldots
$$

Note that $0<a_{n+1} \leq a_{n}<\infty$ for each $n$. Thus the limit $a=\lim _{n \rightarrow \infty} a_{n}$ exists. We divide the proof into two parts.

(i) Suppose $a>0$. Then there exists a sequence $t_{n} \rightarrow 0^{+}$such that $\phi\left(t_{n}\right) x^{*}\left(t_{n}\right) \geq a / 2$ for each $n$. Thus

$$
\left\|x^{*} \chi_{\left(0, t_{n}\right)}\right\|_{L^{\infty}(\phi)}=\sup _{t \in\left(0, t_{n}\right]} x^{*}(t) \phi(t) \geq a / 2
$$

Consequently, by Lemma 4.7, $x$ is not an $H_{g}$-point of $M_{\phi}^{(*)}$.

(ii) Assume that $a=0$. Set $C=\left\{t:|x(t)|>x^{*}(\infty)\right\}$. We consider two subcases.

(1) Suppose $m(C)>0$. Then, by Lemma 2.2 from [7], there is a measure preserving transformation $\sigma: C \rightarrow(0, m(C))$ such that $x^{*} \circ \sigma=|x|$ a.e. on $C$. Since $a=0$ and $x \neq 0$, there is $0<\delta<m(C)$ such that

$$
\sup _{t \in(0, \delta]} x^{*}(t) \phi(t)<\frac{1}{2}\|x\|_{M_{\phi}^{(*)}}
$$

whence $\|x\|_{M_{\phi}^{(*)}} \geq 2 \phi(t) x^{*}(t)$ for $t \leq \delta$. Let $\left(A_{n}\right)$ be the sequence of measurable sets such that $A_{n}=\sigma^{-1}((0, \delta / n)) \subset C$. Then

$$
\|x\|_{M_{\phi}^{(*)}} \geq 2 \phi(\sigma(s)) x^{*}(\sigma(s))
$$

for $s \in A_{n}$. Denote

$$
\widetilde{\phi_{n}}(s)=\phi(\sigma(s)) \text { for } s \in A_{n} \text { and } x_{n}=\frac{\|x\|_{M_{\phi}^{(*)}}}{\widetilde{\phi}_{n}} \chi_{A_{n}}+x \chi_{I \backslash A_{n}} .
$$

Then $x_{n} \rightarrow x$ globally in measure. Note also that

$$
\begin{aligned}
& \sup _{t \in(0, \delta / n]}\left(\frac{\|x\|_{M_{\phi}^{(*)}}}{\widetilde{\phi}_{n}} \chi_{A_{n}}+x \chi_{I \backslash A_{n}}\right)^{*}(t) \phi(t) \\
& =\sup _{t \in(0, \delta / n]} \frac{\|x\|_{M_{\phi}^{(*)}}}{\phi(t)} \chi_{(0, \delta / n]}(t) \phi(t)=\|x\|_{M_{\phi}^{(*)}}
\end{aligned}
$$


and, by (5.1),

$\sup _{t>\delta / n}\left(\frac{\|x\|_{M_{\phi}^{(*)}}}{\widetilde{\phi}_{n}} \chi_{A_{n}}+x \chi_{I \backslash A_{n}}\right)^{*}(t) \phi(t)=\sup _{t>\delta / n} x^{*}(t) \chi(\delta / n, \infty)(t) \phi(t)=\|x\|_{M_{\phi}^{(*)}}$,

whence $\left\|x_{n}\right\|_{M_{\phi}^{(*)}}=\|x\|_{M_{\phi}^{(*)}}$. Notice that

$$
\begin{aligned}
\frac{\|x\|_{M_{\phi}^{(*)}}}{\widetilde{\phi_{n}}(s)}-x(s) & \geq \frac{\|x\|_{M_{\phi}^{(*)}}}{\widetilde{\phi_{n}}(s)}-|x(s)|=\frac{\|x\|_{M_{\phi}^{(*)}}}{\phi(\sigma(s))}-x^{*}(\sigma(s)) \\
& \geq \frac{\|x\|_{M_{\phi}^{(*)}}}{\phi(\sigma(s))}-\frac{\|x\|_{M_{\phi}^{(*)}}}{2 \phi(\sigma(s))}=\frac{\|x\|_{M_{\phi}^{(*)}}}{2 \widetilde{\phi}_{n}(s)}
\end{aligned}
$$

for $s \in A_{n}$. Therefore, applying the equality $m\left(A_{n}\right)=m\left(\sigma^{-1}((0, \delta / n))\right)=$ $m((0, \delta / n))$, we get

$$
\begin{aligned}
\left\|x_{n}-x\right\|_{M_{\phi}^{(*)}} & =\sup _{t>0}\left(x_{n}-x\right)^{*}(t) \phi(t)=\sup _{t \in(0, \delta / n]}\left(\frac{\|x\|_{M_{\phi}^{(*)}}}{\widetilde{\phi}_{n}} \chi_{A_{n}}-x \chi_{A_{n}}\right)^{*}(t) \phi(t) \\
& \geq \sup _{t \in(0, \delta / n]}\left(\frac{\|x\|_{M_{\phi}^{(*)}}}{2 \widetilde{\phi_{n}}} \chi_{A_{n}}\right)^{*}(t) \phi(t)=\frac{\|x\|_{M_{\phi}^{(*)}}}{2} .
\end{aligned}
$$

(2) Assume that $m(C)=0$. Then $x^{*}=b \chi_{(0, \infty)}$ for some $b>0$, because $x \neq 0$. Since $a=0$, we find a number $\delta>0$ such that

$$
\sup _{t \in(0, \delta]} x^{*}(t) \phi(t)=\sup _{t \in(0, \delta]} b \phi(t)<\frac{1}{2}\|x\|_{M_{\phi}^{(*)}} .
$$

Taking a sequence of sets $A_{n}=(0, \delta / n)$ and

$$
x_{n}=\frac{\|x\|_{M_{\phi}^{(*)}}}{\phi} \chi_{A_{n}}+x \chi_{I \backslash A_{n}}
$$

we finish as above.

\section{Kadec-Klee properties in Lorentz spaces}

Here we consider the Lorentz spaces $\Lambda_{\phi}$ defined in Sect. 4 and also the Lorentz spaces $\Lambda_{p, w}, 0<p<\infty$, defined by the norm

$$
\|x\|_{p, w}=\left(\int_{I}\left(x^{*}\right)^{p} w\right)^{1 / p}
$$


where $w: I \rightarrow(0, \infty)$ is a measurable weight function satisfying the condition $W(t):=\int_{0}^{t} w<\infty$ for all $t \in I$. Then $\Lambda_{p, w}=\left(L_{p}\left(w^{1 / p}\right)\right)^{(*)}$. Moreover, $\|\cdot\|_{p, w}$ is a quasi-norm (equivalently $\Lambda_{p, w}$ is a linear space) if and only if $W$ satisfies condition $\Delta_{2}$, that is there is a constant $K>0$ such that $W(2 t) \leq K W(t)$ for all $t \in \frac{1}{2} I$ (see $[9,20])$. Consequently, we assume always that $W \in \Delta_{2}$. It is also known that $\|\cdot\|_{p, w}$ is a norm if and only if $p \geq 1$ and $w$ is nonincreasing. Note that if $w$ is nonincreasing then $\Lambda_{1, w}$ is a particular case of $\Lambda_{\phi}$ when $\phi\left(0^{+}\right)=0$.

Corollary 6.1 Let $p>0$. The space $\Lambda_{p, w}$ has the property $H_{g}$ that is each point $x \in \Lambda_{p, w}$ is an $H_{g}$-point.

Proof We apply Theorem 4.9, the implication (i) $\Rightarrow$ (ii) with $E=L_{p}\left(w^{1 / p}\right)$. Clearly, $L_{p}\left(w^{1 / p}\right)$ is $p$-convex with the constant 1 . Note that $L_{p}\left(w^{1 / p}\right) \in(O C)$, whence $x^{*} \in(*)$ for each $x^{*} \in L_{p}\left(w^{1 / p}\right)$ (see Example 4.8(i)). Let $x \in \Lambda_{p, w} \backslash\{0\}$. Then $x^{*} \in L_{p}\left(w^{1 / p}\right)$. Take a sequence $a_{n} \rightarrow 0$. Since $L_{p}\left(w^{1 / p}\right) \in(O C)$, we have $\left\|x^{*} \chi_{\left(0, a_{n}\right)}\right\|_{L_{p}\left(w^{1 / p}\right)} \rightarrow 0$.

Now we prove that $x^{*}$ is an $H_{g}^{+}$-point of $E=L_{p}\left(w^{1 / p}\right)$ which is stronger than $H_{g}^{*}$-point of $E$. In view of Theorem 3.15 we divide the proof in two parts.

(i) Let $\left(d_{n}\right) \subset E_{+}$be a sequence such that $d_{n} \leq x^{*}$ and $d_{n} \rightarrow 0$ globally in measure. Then, by $L_{p}\left(w^{1 / p}\right) \in(O C)$, we conclude that $\left\|d_{n}\right\|_{E} \rightarrow 0$.

(ii) Recall that a quasi-Banach function space $E$ is said to be uniformly monotone $\left(E \in(U M)\right.$ briefly) if for all sequences $0 \leq y_{n} \leq x_{n} \in E$ satisfying $\left\|x_{n}\right\|_{E}-\left\|y_{n}\right\|_{E} \rightarrow 0$ we have $\left\|x_{n}-y_{n}\right\|_{E} \rightarrow 0$ (see [4]). Consider a sequence $\left(y_{n}\right) \subset E_{+}$such that $x^{*} \leq y_{n}, y_{n} \rightarrow x^{*}$ globally in measure and $\left\|y_{n}\right\|_{E} \rightarrow\left\|x^{*}\right\|_{E}$. Thus $\left\|y_{n}-x^{*}\right\|_{E} \rightarrow 0$, because $L_{p}\left(w^{1 / p}\right) \in(U M)$. Indeed, by the definition, $L_{1}(w) \in(U M)$. Moreover, $\left(L_{1}(w)\right)^{(p)} \in(U M)$, by Corollary 4.5 from [30] (note that $L_{1}(w)$ satisfies the respective assumption). Since $\left(L_{1}(w)\right)^{(p)} \equiv$ $L_{p}\left(w^{1 / p}\right)$, the proof is finished.

Remark 6.2 For $p \geq 1$, the previous Corollary can be concluded also from [23, Corollary 3.21] (with a different proof).

Consider the Lorentz space $\Lambda_{\phi}=\left(L^{1}(d \phi)\right)^{(*)}$ with $\phi$ being concave. If $\phi\left(0^{+}\right)=0$ then $\Lambda_{\phi}=\left(L^{1}\left(\phi^{\prime}\right)\right)^{(*)}$. Recall that

$$
\|x\|_{L^{1}(d \phi)}=\phi\left(0^{+}\right)\|x\|_{L^{\infty}(I)}+\int_{I}|x(t)| \phi^{\prime}(t) d t .
$$

Remark 6.3 (i) If $\phi\left(0^{+}\right)>0$ or $\phi^{\prime}>0$ then $\|\cdot\|_{L^{1}(d \phi)}$ is a norm. If $\phi\left(0^{+}\right)=0$ and $\phi^{\prime}(t)=0$ for $t \geq a \in(0, m(\mathrm{I}))$ then $\|\cdot\|_{L^{1}(d \phi)}$ is only a semi-norm (it satisfies the triangle inequality, it is homogeneous and $\left.\|0\|_{L^{1}(d \phi)}=0\right)$ but still $L^{1}(d \phi)^{(*)}$ is a normed space. 
(ii) Note that $L^{1}(d \phi) \in(+)$. Indeed, by Hardy-Littlewood inequality,

$$
\begin{aligned}
\|x\|_{L^{1}(d \phi)}= & \phi\left(0^{+}\right)\|x\|_{L^{\infty}(I)}+\int_{I}|x(t)| \phi^{\prime}(t) d t \leq \phi\left(0^{+}\right)\left\|x^{*}\right\|_{L^{\infty}(I)} \\
& +\int_{I} x^{*}(t) \phi^{\prime}(t) d t=\left\|x^{*}\right\|_{L^{1}(d \phi)},
\end{aligned}
$$

in view of the fact that $\phi^{\prime}$ is nonincreasing (see [3,29]).

Note that the point (ii) below has been proved directly in [6, Corollary 1.3], see also [23, Corollary 3.21].

Corollary 6.4 (i) If $\phi\left(0^{+}\right)>0$ then $\Lambda_{\phi}$ has no $H_{g}$-points.

(ii) Suppose $\phi\left(0^{+}\right)=0$. Then $\Lambda_{\phi} \in\left(H_{g}\right)$ that is each $x \in \Lambda_{\phi}$ is an $H_{g}$-point.

Proof (i) Let $0 \neq x \in \Lambda_{\phi}$. Then there is a number $\delta>0$ such that the set $A=$ $\{t \in I:|x(t)| \geq \delta\}$ has a positive measure. Take a sequence $\left(A_{n}\right)$ in $A$ with $0<$ $m\left(A_{n}\right) \rightarrow 0$. Let $x_{n}=|x| \chi_{A_{n}}$. Then $x_{n} \rightarrow 0$ globally in measure and

$$
\left\|x_{n}\right\|_{\Lambda_{\phi}} \geq \phi\left(0^{+}\right)\left\|x_{n}^{*}\right\|_{L^{\infty}(I)} \geq \phi\left(0^{+}\right) \delta .
$$

By Lemma 3.14, $x$ is not an $H_{g}$-point. Clearly, the space $\Lambda_{\phi}$ satisfies assumptions of Lemma 3.14 because it is just a normed space.

(ii) We follow as in the proof of Corollary 6.1. Note that Theorem 4.9, the implication (i) $\Rightarrow$ (ii), is true also if $(E,\|\cdot\|)$ is only a semi-quasi normed space.

Remark 6.5 The case $\phi\left(0^{+}\right)=0$ and $\phi^{\prime}>0$ above is included in the Corollary 6.1.

Obviously, the notions of $H_{g}$-point and $H_{l}$-point coincide if $I=(0,1)$. Consequently, we consider only the case $I=(0, \infty)$ below.

Corollary 6.6 (i) Suppose $p \geq 1$ and $w$ is nonincreasing. The element $0 \leq x \in \Lambda_{p, w}$ is an $H_{l}$-point of $\Lambda_{p, w}$ if and only if $x^{*}(\infty)=0$.

(ii) Assume that $\phi\left(0^{+}\right)=0$. The element $0 \leq x \in \Lambda_{\phi}$ is an $H_{l}$-point of $\Lambda_{\phi}$ if and only if $x^{*}(\infty)=0$ and $m\left\{t \in I: x^{*}(t)<x^{*}\left(\gamma^{-}\right)\right\}=0$ whenever $\gamma<\infty$ with $\gamma=m\left(\operatorname{supp} \phi^{\prime}\right)$.

(iii) If $\phi\left(0^{+}\right)>0$ then $\Lambda_{\phi}$ has no $H_{l}$-points.

Proof (i) By assumptions, $\Lambda_{p, w}$ is a symmetric Banach space. By Theorem 3.10 from [8], $x \in \Lambda_{p, w}$ is an $H_{l}$-point of $\Lambda_{p, w}$ if and only if $x \in\left(\Lambda_{p, w}\right)_{a}, x$ is an $H_{g}$-point of $\Lambda_{p, w}$ and $x$ is a $U M$-point of $\Lambda_{p, w}$. Next, $x \in\left(\Lambda_{p, w}\right)_{a}$ if and only if $x^{*}(\infty)=0$ (the necessity follows from Lemma 3.1, the sufficiency from Lebesgue dominated convergence theorem). Moreover, each $x \in \Lambda_{p, w}$ is an $H_{g}$-point by Corollary 6.1. Finally, $x$ is a $U M$-point of $\Lambda_{p, w}$ if and only if $x^{*}$ is a $U M^{*}$-point of $L_{p}\left(w^{1 / p}\right)$ and $m\left\{t \in I: x^{*}(t)<x^{*}(\infty)\right\}=0$ by Theorem 3.8 in [25]. The condition $x^{*}(\infty)=0$ gives automatically that $m\left\{t \in I: x^{*}(t)<x^{*}(\infty)\right\}=0$. Finally, each $x^{*}$ is a $U M^{*}$ point of $L_{p}\left(w^{1 / p}\right)$, because $w>0$. 
(ii) Obviously, $\Lambda_{\phi}$ is a symmetric Banach space. By Theorem 3.10 from [8], $x \in \Lambda_{\phi}$ is an $H_{l}$-point of $\Lambda_{\phi}$ if and only if $x \in\left(\Lambda_{\phi}\right)_{a}, x$ is an $H_{g}$-point of $\Lambda_{\phi}$, and $x$ is a $U M$-point of $\Lambda_{\phi}$. Next, $x \in\left(\Lambda_{\phi}\right)_{a}$ if and only if $x^{*}(\infty)=0$ (see for example Corollary 4.13 in [25]). Moreover, each $x \in \Lambda_{\phi}$ is an $H_{g}$-point by Corollary 6.4. Finally, by Corollary 4.17 in [25], if $x^{*}(\infty)=0$ then $x$ is a $U M$-point of $\Lambda_{\phi}$ if and only if $m\left\{t \in I: x^{*}(t)<x^{*}\left(\gamma^{-}\right)\right\}=0$ whenever $\gamma<\infty$ with $\gamma=m\left(\operatorname{supp} \phi^{\prime}\right)$.

(iii) It follows from Corollary 6.4.

\section{Questions}

1. The full characterization of an $H_{l}$-point in a symmetric Banach function space on $I=(0, \infty)$ has been given in [8, Theorem 3.10]. Namely, $x$ is an $H_{l}$-point if and only if $x \in E_{a}, x$ is a $U M$-point and $x$ is an $H_{g}$-point. Does it remain true for a symmetric quasi-Banach function space? If yes, we can get immediately the characterization of an $H_{l}$-point in symmetrizations $E^{(*)}$ which would be more general than in Corollary 4.16. Unfortunately, one crucial assumption in the proof of Theorem 3.10 in [8] is the embedding $E \hookrightarrow L^{1}+L^{\infty}$ which is not satisfied in general if $E$ is symmetric quasi-Banach function space (see also (2.4)). Although there are examples of symmetric quasi-Banach function spaces with $E \hookrightarrow L^{1}+L^{\infty}$, it seems natural to look for such characterization of $H_{l}$-point without this restricted assumption. Thus new methods should be applied.

2. Recall that $x$ is called $*$-regular if $m\left(\left\{t \in \operatorname{supp} x:|x(t)|<x^{*}(\alpha)\right\}\right)=0$, where $\alpha=m$ (I) (in particular this is the case when $x^{*}(\infty)=0$ or $I=(0,1)$ )—see [8]. Does the implication (ii) $\Rightarrow$ (i) (b) in Theorem 4.9 remain true if we replace the assumption $x^{*}(\infty)=0$ by a weaker one that $x$ is $*$-regular. Perhaps it is possible, because we may apply similar techniques as in the proof of Theorem 3.3 in [8], but someone need to check carefully all details because the case of quasi-normed space is much more delicate than the case of normed space (especially if we consider properties invariant under isometry).

Acknowledgements The author is greatly indebted to the Referee for many valuable comments and helpful suggestions essentially improving the article. The author is supported by the Ministry of Science and Higher Education of Poland, Grant Number 04/43/DSPB/0094.

Open Access This article is distributed under the terms of the Creative Commons Attribution 4.0 International License (http://creativecommons.org/licenses/by/4.0/), which permits unrestricted use, distribution, and reproduction in any medium, provided you give appropriate credit to the original author(s) and the source, provide a link to the Creative Commons license, and indicate if changes were made.

\section{References}

1. Aliprantis, C., Burkinshaw, O.: Locally Solid Riesz Spaces. Pure and Applied Mathematics Series, vol. 76. Academic Press, New York (1978)

2. Bastero, J., Hudzik, H., Steinberg, A.M.: On smallest and largest spaces among rearrangement-invariant $p$-Banach function spaces $(0<p<1)$. Indag. Math. N.S. 2(3), 283-288 (1991)

3. Bennett, C., Sharpley, R.: Interpolation of Operators. Pure and Applied Mathematics Series, vol. 129. Academic Press, New York (1988) 
4. Birkhoff, G.: Lattice Theory. American Mathematical Society, Providence (1967)

5. Cerdà, J., Hudzik, H., Kamińska, A., Mastyło, M.: Geometric properties of symmetric spaces with applications to Orlicz-Lorentz spaces. Positivity 2, 311-337 (1998)

6. Chilin, V.I., Dods, P.G., Sedaev, A.A., Sukochev, F.A.: Characterizations of Kadec-Klee properties in symmetric spaces of measurable functions. Trans. Am. Math. Soc. 348(12), 4895-4918 (1996)

7. Ciesielski, M., Kolwicz, P., Panfil, A.: Local monotonicity structure of symmetric spaces with applications. J. Math. Anal. Appl. 409, 649-662 (2014)

8. Ciesielski, M., Kolwicz, P., Płuciennik, R.: Local approach to Kadec-Klee properties in symmetric function spaces. J. Math. Anal. Appl. 426, 700-726 (2015)

9. Cwikel, M., Kamińska, A., Maligranda, L., Pick, L.: Are generalized Lorentz spaces really spaces? Proc. Am. Math. Soc. 132(12), 3615-3625 (2004)

10. Czerwińska, M.M., Kamińska, A.: Complex rotundities and midpoint local uniform rotundity in symmetric spaces of measurable operators. Stud. Math. 201(3), 253-285 (2010)

11. Dominguez, T., Hudzik, H., Lopez, G., Mastyło, M., Sims, B.: Complete characterization of KadecKlee properties in Orlicz spaces. Houst. J. Math. 29(4), 1027-1044 (2003)

12. Foralewski, P.: Some fundamental geometric and topological properties of generalized Orlicz-Lorentz function spaces. Math. Nachr. 284(8-9), 1003-1023 (2011)

13. Foralewski, P.: On some geometric properties of generalized Orlicz-Lorentz function spaces. Nonlinear Anal. 75(17), 6217-6236 (2012)

14. Hudzik, H., Kolwicz, P., Narloch, A.: Local rotundity structure of Calderón-Lozanovskiŭ spaces. Indag. Math. N.S. 17(3), 373-395 (2006)

15. Hudzik, H., Narloch, A.: Local monotonicity structure of Cardelón-Lozanowskiı̌ spaces. Indag. Math. N.S. 15(1), 1-12 (2004)

16. Kalton, N.J.: Convexity conditions on non locally-convex lattices. Glasg. Math. J. 25, 141-152 (1984)

17. Kalton, N.J., Peck, N.T., Roberts, J.W.: An F-Space Sampler. London Mathematical Society Lecture Note Series. Cambridge University Press, Cambridge (1984)

18. Kantorovich, L.V., Akilov, G.P.: Functional Analysis. Nauka, Moscow (1984). (in Russian)

19. Kamińska, A.: Extreme points in Orlicz-Lorentz spaces. Arch. Math. (Basel) 55(2), 173-180 (1990)

20. Kamińska, A., Maligranda, L.: Order convexity and concavity in Lorentz spaces with arbitrary weight. Stud. Math 160(3), 267-286 (2004)

21. Kamińska, A., Mastyło, M.: Abstract duality Sawyer formula and its applications. Monatsh. Math. 151, 223-245 (2007)

22. Kamińska, A., Raynaud, Y.: Isomorphic copies in the lattice $E$ and its symmetrization $E^{(*)}$ with applications to Orlicz-Lorentz spaces. J. Funct. Anal. 257, 271-331 (2009)

23. Kolwicz, P.: Kadec-Klee properties of Calderó n-Lozanovskiǔ function spaces. J. Funct. Spaces Appl. (2012). https://doi.org/10.1155/2012/314068

24. Kolwicz, P.: Local structure of generalized Orlicz-Lorentz function spaces. Commun. Math. 55(2), 211-227 (2015)

25. Kolwicz, P.: Local structure of symmetrizations $E^{(*)}$ with applications. J. Math. Anal. Appl. 440, 810-822 (2016)

26. Kolwicz, P., Leśnik, K., Maligranda, L.: Pointwise products of some Banach function spaces and factorization. J. Funct. Anal. 266(2), 616-659 (2014)

27. Kolwicz, P., Panfil, A.: Local $\Delta_{2}^{E}$ condition in generalized Calderón-Lozanovskiǔ spaces. Taiwan. J. Math. 16(1), 259-282 (2012)

28. Kolwicz, P., Panfil, A.: Points of nonsquareness of Lorentz spaces $\Gamma_{p, w}$. J. Ineq. Appl. 2014, 467 (2014). https://doi.org/10.1186/1029-242X-2014-467

29. Krein, S.G., Petunin, YuI, Semenov, E.M.: Interpolation of Linear Operators. Nauka, Moscow (1978). (in Russian)

30. Lee, H.J.: Complex convexity and monotonicity in quasi-Banach lattices. Israel J. Math. 159, 57-91 (2007). https://doi.org/10.1007/s11856-007-0038-2

31. Lindenstrauss, J., Tzafriri, L.: Classical Banach Spaces. II. Function Spaces. Springer, Berlin (1979)

32. Maligranda, L.: Type, cotype and convexity properties of quasi-Banach spaces. In: Kato, M., Maligranda, L. (eds.) Banach and Function Spaces. Proceedings of the International Symposium on Banach and Function Spaces (Oct 2-4, 2003, Kitakyushu, Japan), pp. 83-120. Yokohama Publishers (2004)

33. Maligranda, L.: Tosio Aoki (1910-1989). In: Proceedings of the International Symposium on Banach and Function Spaces II Kitakyushu, Japan, pp. 1-23 (2006) 
34. Shimogaki, T.: On the complete continuity of operators in an interpolation theorem. J. Fac. Sci. Hokkaido Univ. Ser. I 20(3), 109-114 (1968)

35. Wnuk, W.: Banach Lattices with Order Continuous Norms. Polish Scientific Publishers PWN, Warszawa (1999) 